\title{
Application of input to state stability to reservoir models
}

\author{
Markus Müller $^{1}(\mathbb{D})$ Carlos A. Sierra ${ }^{1}(\mathbb{D}$
}

Received: 11 November 2016 / Accepted: 31 July 2017 / Published online: 29 August 2017

(C) The Author(s) 2017. This article is an open access publication

\begin{abstract}
Reservoir models play an important role in representing fluxes of matter and energy in ecological systems and are the basis of most models in biogeochemistry. These models are commonly used to study the effects of environmental change on the cycling of biogeochemical elements and to predict potential transitions of ecosystems to alternative states. To study critical regime changes of time-dependent, externally forced biogeochemical systems, we analyze the behavior of reservoir models typical for element cycling (e.g., terrestrial carbon cycle) with respect to time-varying signals by applying the mathematical concept of input to state stability (ISS). In particular, we discuss ISS as a generalization of preceding stability notions for non-autonomous, non-linear reservoir models represented by systems of ordinary differential equations explicitly dependent on time and a time-varying input signal. We also show how ISS enhances existing stability concepts, previously only available for linear time variant (LTV) systems, to a tool applicable also in the non-linear case.
\end{abstract}

Keywords Dynamic stability · Biogeochemical models . Element cycling · Soil organic matter dynamics · ISS . Non-autonomous systems · Time-varying inputs

Markus Müller

mamueller@bgc-jena.mpg.de

Carlos A. Sierra

csierra@bgc-jena.mpg.de

1 Max Planck Institute for Biogeochemistry, 07745 Jena, Germany

\section{Introduction}

\section{Overview}

Since ecological systems may undergo critical transitions that lead to catastrophic regime changes (Scheffer et al. 2001; Beisner et al. 2003; Scheffer and Carpenter 2003; Lenton et al. 2008), it is important to classify mathematical models with respect to the possibility of such transitions. Mathematically, this is achieved through what is commonly referred to as stability analysis (e.g., Guckenheimer and Holmes (1983) and Strogatz (1994)). There is a long history in the ecological sciences concerning the study and use of stability concepts (e.g., May (1973), Holling (1973), Ludwig et al. (1978), and Pimm (1984)), which has lead to considerable confusion about the meaning of the term "stability" itself (Grimm and Wissel 1997). One particular aspect of this confusion can be traced back to the supposed sharp distinction between the concept of stability and that of resilience, which was introduced more than four decades ago by Holling (1973). While we do not want to discuss the complex history of the notion of resilience here, we do want to point out the possible effect of its original definition unduly narrowing the perceived focus of stability analysis as restricted to autonomous systems close to equilibria only. In a recent review, Meyer (2016) discusses the concept of resilience exclusively in the light of autonomous systems. In fact, most of the mathematical tools that have previously been used to study stability in ecological systems rely heavily on restrictions imposed by the assumption of an autonomous system. This raises the following question: Do ecologically meaningful stability concepts even exist if the system is

1. Non-autonomous, i.e., explicitly dependent on time 
2. Not in equilibrium, because continually disturbed by a time-dependent driving signal $\mathbf{u}(t)$ :

$\dot{\mathbf{x}}=\mathbf{h}(t, \mathbf{x}, \mathbf{u}(t))$,

where $\mathbf{x}$ describes the state of the system and $\dot{\mathbf{x}}$ its derivative with respect to time expressed by the vector valued function $\mathbf{h}$ of time $t$, state $\mathbf{x}$, and a driving signal $\mathbf{u}(t)$.

We review here the concept of input to state stability (ISS) recently developed in control theory (Sontag 2008) as one such classifying concept by applying it to element cycling reservoir systems where the $i$ th component of $\mathbf{x}$ will usually denote the content of pool $i$. In particular, we will show the following: (a) why a tool able to deal with conditions 1 and 2 is not only useful but essential for many ecological models, (b) what kind of behavior the ISS systems exhibit, (c) how the ISS property can be proved for a non-linear example system, and (d) how ISS naturally generalizes stability notions previously used in ecology for both autonomous non-linear and linear time-varying systems, thereby revealing a large number of implicit applications.

ISS is not the only concept in this area. Alternatives include the mathematically more general theory of nonautonomous systems (Kloeden and Rasmussen 2011) and much more concrete examples and applications like the "compost bomb" (Wieczorek et al. 2011). Probabilistic approaches have also been proposed (Nolting and Abbott 2016). We will be able to point out some connections after ISS has been properly introduced.

To reach ecologists, we discuss concrete examples in a general way. To satisfy the more mathematically oriented, we underpin these claims by proofs. This dichotomy is reflected in the structure. In the paper, we give an overview and try to draw the bigger picture. Many of the detailed definitions, explanations, and actual technical results substantiating it have been included in the appendices.

\section{Motivation}

Note that in the past, many ecological models have been formulated as autonomous systems of ordinary differential equations (ODEs) of the form

$\dot{\mathbf{x}}=\mathbf{f}(\mathbf{x}, \mathbf{p})$,

where $\dot{\mathbf{x}}=\frac{d \mathbf{x}}{d t}$ is the derivative with respect to time and $\mathbf{p}$ is a set of parameters assumed to be fixed (MaynardSmith 1978; Kot 2001; Cushing et al. 2003; Pastor 2008; Soetaert and Herman 2009). These autonomous systems are a proper subset of dynamical systems, their dynamics consisting solely in their ability to capture the influence of $\mathbf{x}$ on its own rate of change $\dot{\mathbf{x}}$. If one looks now for invari- ant sets such as fixed points and conditions ensuring their stability, one can do so with respect to the dynamic character of $\mathbf{x}$ only. Although stability or existence of invariant sets can be discussed in view of changing parameters (e.g., in bifurcation analysis), the theory of autonomous systems does not provide tools to capture the influence of the timedependent nature (for instance the speed) of such parameter changes. As a remedy, the parameters $\mathbf{p}$ in system (2) are sometimes assumed to change extremely slowly in comparison to $\mathbf{x}$. However, although very useful when appropriate (for instance Ludwig et al. (1978)), this quasi-static approximation can be dangerously misleading and hard to justify beforehand, as the following example shows. The system

$\dot{\mathbf{x}}=\mathbf{A}(p) \mathbf{x}$

with

$\mathbf{A}(p)=\left(\begin{array}{ll}-1+1.5 \cos ^{2} p & 1-1.5 \sin p \cos p \\ -1-1.5 \sin p \cos p-1+1.5 \sin ^{2} p\end{array}\right)$

has a stable fixed point at $\mathbf{0}$ which is stable for all $p \in \mathbb{R}$ since the eigenvalues of $\mathbf{A}(p)$, given by $\lambda_{1,2}=\frac{-1 \pm i \sqrt{7}}{4}$, have a negative real part. Nevertheless, the solution for the time-dependent $p(t)=t$ given by

$\mathbf{x}=\Phi(t) \mathbf{x}_{0}$,

with

$\Phi(t)=\left(\begin{array}{ll}e^{0.5 t} \cos t & e^{-t} \sin t \\ -e^{0.5 t} \sin t & e^{-t} \cos t\end{array}\right)$,

will in general not even converge to a fixed point (except when $x_{1}(0)=0$ ), but will instead grow exponentially with time. This extremely unstable behavior would remain for any $k$ in $p(t)=\frac{t}{k}$. To avoid surprises like this, we have to consider stability notions for explicitly time-dependent systems.

Many models in ecosystem ecology and biogeochemistry use time-dependent functions to modify parameters related to environmental effects: e.g., photosynthetic carbon fixation or organic matter inputs to soils (Luo and Weng 2011; Raupach 2013; Luo et al. 2015; Sierra and Müller 2015). Capturing only the internal (state induced) dynamics of these models is obviously insufficient for stability analysis, and they must instead be represented as non-autonomous dynamical systems:

$$
\begin{aligned}
\dot{\mathbf{x}} & =\mathbf{f}(\mathbf{x}, \mathbf{p}(t)) \\
& =\mathbf{g}(\mathbf{x}, t) .
\end{aligned}
$$

We can look at this situation from two different perspectives. On one hand, if we are still interested in invariant sets, Eq. 6 indicates that we now must explicitly consider the possibility that they vanish due to the time dependency. Instead of merely ignoring this possibility, we now have to check, 
that it does not occur. For example, systems of form (6) may still have fixed points, but it is no longer enough to show this for (even arbitrarily chosen) fixed times. On the other hand, we do not always expect fixed points, but are instead interested in how the system responds to a time-dependent driving signal $\mathbf{p}(t)$ as suggested by Eq. 5 .

Combining both views, we can choose some components of $\mathbf{p}(t)$ as a driving signal $\mathbf{u}(t)$ and absorb others in the definition of $\mathbf{h}$ in Eq. 1. For instance, in discussing effects of time-dependent environmental variability expressed by temperature $T(t)$, precipitation $P(t)$, and photosynthetic carbon fixation $F(t)$ on the carbon stocks $C$ of an ecosystem, we could choose $\mathbf{p}(t)$ in Eq. 5 as $\mathbf{p}(t)=(T(t), P(t), F(t))$. We could treat any single term or any combinations of terms $\{T, P, F\}$ as a driving signal and represent the others by the explicit $t$ dependency of $\mathbf{h}$. As the perhaps simplest concrete example, imagine a single-pool, linear ecosystem model

$\dot{C}=F(t)-k(T(t), P(t)) C$

with decomposition/respiration rate in product form $k(T, P)=k_{0} T P$ and some possible choices of $\mathbf{u}$. We can choose a three-dimensional driver, for instance $\mathbf{u}(t)=$ $\left(F(t)-F_{0}, T(t)-T_{0}, P(t)-P_{0}\right)$ where $F_{0}, T_{0}$, and $P_{0}$ denote some constants. This implies that $h(\mathbf{u}, t)=h(\mathbf{u})=$ $u_{1}+F_{0}+k_{0}\left(T_{0}+u_{2}\right)\left(P_{0}+u_{3}\right)$ depends on $t$ only through $\mathbf{u}$. We could also choose two-dimensional signals, for instance $\mathbf{u}(t)=\left(T(t)-T_{0}, P(t)-P_{0}\right)$ resulting in the explicitly time-dependent $h(\mathbf{u}, t)=F(t)+k_{0}\left(u_{1}+T_{0}\right)\left(u_{2}+P_{0}\right)$. Of course, $\mathbf{u}(t)=\left(F(t)-F_{0}, P(t)-P_{0}\right)$, and $\mathbf{u}(t)=$ $\left(F(t)-F_{0}, T-T_{0}\right)$ are also possible as well as onedimensional definitions like $u(t)=F(t)-F_{0}$ resulting in $h(u, t)=u-k_{0} P(t) T(t)$, or $u(t)=T(t)-T_{0}$, or $u(t)=P(t)-P_{0}$.

Another source for driving signals $\mathbf{u}$ is uncertainty in some parameter. We will see that the ISS framework does not require smoothness of $\mathbf{u}$, which makes it possible to include stochastic signals, which are, like Brownian motion, frequently not smooth.

In ecological reservoir systems, so far only special cases of Eq. 1 have been discussed, with very different concepts. For example, Manzoni et al. (2009), although not concerned with stability directly, applied linear input response theory. In control theory, the linear response approach can be seen as a less general predecessor to ISS for linear time invariant (LTI) systems described by an equation of the form (5) with f linear in x. Rasmussen et al. (2016) consider stability of linear time-varying systems of form (6), but not with respect to a driving signal.

In contrast to this previous work, we will apply the single concept of ISS, which has become important in non-linear control theory in recent years and has also found its way into models of chemical (Chaves 2005) and biological systems (Chaves et al. 2008). We will use a generalized version for time-varying systems. We also show that ISS seamlessly generalizes concepts of stability for LTI and LTV cases.

\section{Definition of ISS}

In the remainder of this section, we will introduce some necessary nomenclature and show how the traditional stability notions for autonomous and non-autonomous dynamical systems are embedded within ISS. Although we decided to present these notions in Appendix 1, a considerable part of our work has been spent to formulate them in a way that especially accommodates their generalization to ISS.

While our derivation in Appendix 1 has concentrated on versions of stability available for autonomous systems and also applicable in the non-autonomous cases, the following text will present extended notions of stability, where fixed points or invariant sets cannot be maintained against the driving signal, but act instead as central locations around which the systems move, with amplitudes controlled by the amplitudes of the driving forces, and to which the systems return when the driving forces disappear. This case is the subject of control theory, where we import the concept of input to state stability from. The great utility of ISS will be demonstrated by showing some examples of the multitude of possible equivalent definitions suitable for different applications.

We divide the presentation into two parts distinguishing between levels of time dependency.

\section{Some nomenclature}

Let $\mathbb{R}^{+}$denote the non-negative real numbers, $\mathbb{R}^{+^{n}}$ the $n$ dimensional, non-negative orthant, and $|\mathbf{x}|_{\mathcal{A}}=\inf _{\mathbf{y} \in \mathcal{A}} \mid \mathbf{x}-$ $\mathbf{y} \mid$ the distance to a set. The disturbances will be measurable locally bounded maps in the space $\mathcal{U}=L^{\infty}\left(\left[t_{0}, \infty\right) ; \mathbb{R}^{m}\right)$

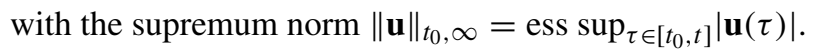

Definition 1 (Forward complete system) Let $\mathbf{x}\left(t, \mathbf{x}_{0}, t_{0}, \mathbf{u}\right)$ denote the trajectory of the system (1) corresponding to the initial condition $\mathbf{x}\left(t_{0}\right)=\mathbf{x}_{0}$ and the input function $\mathbf{u}$. This solution is uniquely defined on some maximum interval $\left[t_{0}, T_{t_{0}, \mathbf{x}_{0}, \mathbf{u}}\right)$. If $T_{t_{0}, \mathbf{x}_{0}, \mathbf{u}}=\infty$, system (1) is called forward complete.

Definition 2 (Invariant set and zero-invariant set) An invariant set $\mathcal{A}$ of a dynamical system (1) is a region of the state space that solutions $\mathbf{x}\left(t, \mathbf{x}_{0}, t_{0}, \mathbf{u}\right)$ will never leave.

$\mathbf{x}_{0} \in \mathcal{A} \Longrightarrow \mathbf{x}\left(t, \mathbf{x}_{0}, t_{0}, \mathbf{u}\right) \in \mathcal{A}$, for all $t \geq t_{0}$.

A zero-invariant set $\mathcal{A}_{0}$ of system (1) is an invariant set of the undisturbed system

$\mathbf{x}_{0} \in \mathcal{A}_{0} \Longrightarrow \mathbf{x}\left(t, \mathbf{x}_{0}, t_{0}, \mathbf{0}\right) \in \mathcal{A}_{0}$, for all $t \geq t_{0}$. 
We will see examples where the minimal zero-invariant set consists of a single fixed point, although the $t$ dependency of Eq. 1 makes this a rather rare case.

Definition $3\left(\mathcal{K}_{\infty}\right.$ and $\mathcal{K} \mathcal{L}$ functions) A class $\mathcal{K}$ function is a function $\alpha: \mathbb{R}^{+} \rightarrow \mathbb{R}^{+}$that is continuous, is strictly increasing, and satisfies $\alpha(0)=0$.

A class $\mathcal{K}_{\infty}$ function is an unbounded class $\mathcal{K}$ function satisfying

$\alpha(r) \nearrow \infty$ as $r \rightarrow \infty$.

A class $\mathcal{K} \mathcal{L}$ function is a function $\beta: \mathbb{R}^{+} \times \mathbb{R}^{+} \rightarrow \mathbb{R}^{+}$ such that

$\beta(\cdot, t) \in \mathcal{K}_{\infty}$ for all $t \quad$ and $\quad \beta(r, t) \searrow 0$ as $t \rightarrow \infty$.

In our examples, solutions of system (1) will develop in $\mathcal{X}=\mathbb{R}^{+^{n}}$ and the invariant sets $\mathcal{A}$ could sometimes touch the boundaries. Therefore, we give an adapted definition of the interior of a set in $\mathcal{X}$ (Chaves et al. 2008).

Definition 4 (Interior of a set $\mathcal{R}$ in $\mathcal{X}$ ) For $\mathcal{R} \subset \mathcal{X}$, we define the interior of $\mathcal{R}$ by

$\operatorname{int}_{\mathcal{X}} \mathcal{R}:=\{\mathbf{x} \in \mathcal{R}: \mathbf{x} \in \operatorname{int}(R)$ or $\mathbf{x} \in \partial \mathcal{R} \cap \partial \mathcal{X}\}$

\section{Definitions of ISS where the time dependency is restricted} to the input signal $\mathbf{u}$ only

We consider in this section a system of the form

$\dot{\mathbf{x}}=\mathbf{h}(\mathbf{x}, \mathbf{u})$

We start with a rather abstract definition of ISS, close to the one most commonly encountered in textbooks, that we will use later to establish the connection to linear systems. Other more illustrating definitions follow.

\section{Remark:}

Let $\mathcal{X}$ denote the domain in which solutions of Eq. 7 develop. (So, by definition, the set $\mathcal{X}$ is also invariant.) In many standard applications of ISS, $\mathcal{X}=\mathbb{R}^{n}$ and $\mathcal{X}$ is not even mentioned. In these cases, the ISS definitions can focus on the smaller set $\mathcal{A}$, which conceptually is the essential invariant set. But when the state variables-as in our examples-describe inherently non-negative masses or concentrations, $\mathcal{X}$ becomes a proper subset of $\mathbb{R}^{n}$, usually $\mathcal{X}=\mathbb{R}^{+^{n}}$. Therefore, the following definition gets a bit more cumbersome by explicit reference to $\mathcal{X}$ and its invariance. Readers yet unfamiliar with ISS are asked not to get distracted by this technical necessity, but to focus on the neighborhood of $\mathcal{A}$ where the typical ISS behavior can be observed.
Definition 5 (ISS) Let $\mathcal{X}$ be an invariant set of system (1). If there exists a set $\mathcal{D} \subset \mathcal{X}$ with $\mathcal{A} \subset$ int $_{\mathcal{X}}(\mathcal{D})$ and a $\mathcal{K} \mathcal{L}$ function $\beta_{\mathcal{D}}$, a class $\mathcal{K}$ function $\gamma_{\mathcal{D}}$, such that

$\left|\mathbf{x}\left(t, \mathbf{x}_{0}, t_{0}, \mathbf{u}\right)\right|_{\mathcal{A}} \leq \beta_{\mathcal{D}}\left(\left|\mathbf{x}_{0}\right|_{\mathcal{A}}, t\right)+\gamma_{\mathcal{D}}\left(\|\mathbf{u}\|_{t_{0}, \infty}\right)$, for all $t \geq 0$

for all $\mathbf{x}_{0} \in \mathcal{D}$ and all inputs $\mathbf{u} \in \mathcal{U}$, then system (1) is said to be locally ISS with respect to $\mathcal{A}$. It is said to be input to state stable, or (globally) ISS, if $\mathcal{D}=\mathcal{X}$ and Eq. 8 is satisfied for any initial state $\mathbf{x}_{0}$, and any input $\mathbf{u} \in \mathcal{U}$.

Note that this local definition already enables the application to multi-stable systems. It has been used to prove ISS for bi-stable biological examples (Chaves et al. 2008) that are very similar in structure to some non-linear biogeochemical models. To interpret it more easily, note that a very simple change leads to the following alternative definition. Since $\max \{a, b\} \leq a+b \leq \max \{2 a, 2 b\}$, Eq. 8 can be replaced by

$\left|\mathbf{x}\left(t, \mathbf{x}_{0}, t_{0}, \mathbf{u}\right)\right|_{\mathcal{A}} \leq \max \left\{\beta\left(\left|\mathbf{x}_{0}\right|_{\mathcal{A}}, t\right), \gamma\left(\|\mathbf{u}\|_{t_{0}, \infty}\right)\right\}$

with different $\gamma$ and $\beta$. In words: for an ISS system, the distance of any solution to the set $\mathcal{A}$ depends monotonically on the original distance of the start value $\left|\mathbf{x}_{0}\right|_{\mathcal{A}}$ and the size of the disturbing input signal $\|\mathbf{u}\|_{t_{0}, \infty}$, where the influence of the start value decreases with time and vanishes asymptotically, so that the distance will be dominated by the input. Remarkably, ISS combines both influences quantitatively.

How the asymptotic behavior of an ISS system depends on start values and inputs is further enlightened by the "non-linear superposition principle" (Sontag et al. 1996); the influences of start value and disturbing signal are "superimposed" as illustrated in Fig. 1. ISS is equivalent to the combination of the following 0-globally asymptotically stable (0-GAS) and "asymptotic gain" (AG) properties:

Definition 6(0-GAS) System (7) is said to be 0-GAS if the input-free system $(\mathbf{u}(t)=\mathbf{0}$ for all $t)$ is GAS, as defined in Appendix 1 for autonomous systems.

Definition 7 (Asymptotic gain property AG) System (7) is said to exhibit the AG property if there exists a $\gamma \in \mathcal{K}_{\infty}$ such that

$\varlimsup_{t \rightarrow+\infty}\left|\mathbf{x}\left(t, \mathbf{x}_{0}, t_{0}, \mathbf{u}\right)\right|_{\mathcal{A}} \leq \gamma\left(\|\mathbf{u}\|_{t_{0}, \infty}\right)$, for all $\mathbf{x}_{0}, \mathbf{u}($.

Theorem 1 (ISS is 0-GAS + AG, Sontag et al. (1996)) $A$ system is ISS if and only if it is $0-G A S$ and AG.

The theorem permits an alternative definition of (global) ISS that is important for showing that ISS naturally 


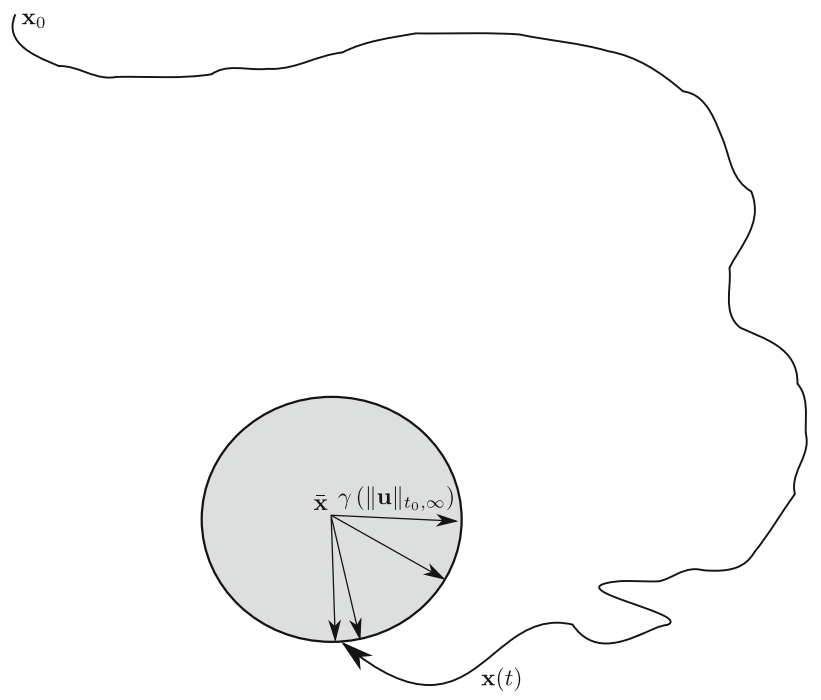

Fig. 1 The asymptotic gain property for a fixed point $\overline{\mathbf{x}}$ (left-hand side) and a zero-invariant set $\mathcal{A}$ (right-hand side). Regardless of the start value $\mathbf{x}_{0}=\mathbf{x}\left(t_{0}\right)$, every solution will eventually converge to the region (light grey) defined by the distance to the fixed point $\overline{\mathbf{x}}$ (or the zero-invariant set $\mathcal{A}$ respectively). In the case of $\overline{\mathbf{x}}$, the region is an $n$-dimensional ball; in the more general case of an $\mathcal{A}$ with more than one point, it depends on the shape of $\mathcal{A}$. This region could be called

encompasses the GAS concept known from the analysis of autonomous systems, and generalizes GAS by incorporation of the AG property, which deals with the inputs.

\section{Remarks:}

Of course, the requirement of global asymptotic stability is not essential and could be weakened in the same way as in Definition 5 to a version appropriate for smaller basins of attraction. (Basin of attraction is defined in Definition 14 in Appendix 1.1.)

The definition can be generalized to greatly extend the class of systems for which ISS theory can be applied (Angeli and Efimov 2015). This generalization is, however, far beyond the scope of the present work.

A third definition of ISS, which is useful in proofs, gives a "dissipation characterization" using ISS Lyapunov functions (Sontag 2008). It transfers the tool of Lyapunov functions, used to prove stability of autonomous systems, to the domain of ISS. We will use a generalized version of it later to prove ISS for one of our examples.

Definition 8 (Storage function) A continuous function $V$ : $\mathbb{R}^{n} \rightarrow \mathbb{R}$ is a storage function if it is positive definite $(V(\mathbf{0})=0$ and $V(\mathbf{x})>0$ for $x \neq \mathbf{0})$ and proper $(V(\mathbf{x}) \rightarrow \infty$ as $|\mathbf{x}| \rightarrow \infty)$. Equivalently, $V$ is a storage function if there exist $\underline{\alpha}, \bar{\alpha} \in \mathcal{K}_{\infty}$ such that $\underline{\alpha}(|\mathbf{x}|) \leq$ $V(\mathbf{x}) \leq \bar{\alpha}(|\mathbf{x}|)$. When $\mathbf{x}$ represents compartmental contents, the domain of $V$ is $\mathbb{R}^{+^{n}}$, but since we sometimes use coordinate transformations, this is not always the case.

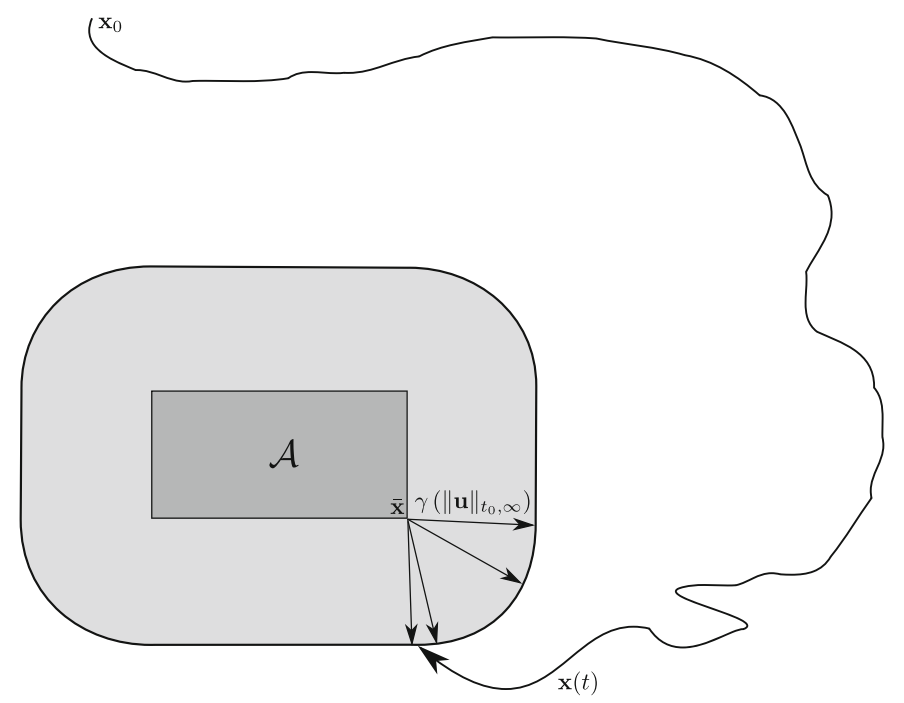

a "region of stability." Its size is limited by the size of the input signal. This distance $\gamma\left(\|\mathbf{u}\|_{t_{0}, \infty}\right)$ is a strictly increasing function $\gamma$ of the supremum norm of the driving signal $\mathbf{u}$. Note that by means of transformations, $\mathbf{u}$ can be seen as the difference to some default input $\mathbf{I}_{d}$ and will be small if $\mathbf{I}$ is close to this default (left-hand side picture inspired by Sontag (2008))

Theorem 2 (Sontag and Wang (1995)) A system is ISS if and only if it admits a smooth ISS Lyapunov function, where an ISS Lyapunov function for Eq. 7 is a smooth storage function $V$ for which there exist functions $\gamma, \alpha \in \mathcal{K}_{\infty}$ such that the following inequality holds:

$\dot{V}(\mathbf{x}, \mathbf{u}) \leq-\alpha(|\mathbf{x}|)+\gamma(|\mathbf{u}|)$

where $\dot{V}=\nabla V \cdot \dot{\mathbf{x}}$ is the orbital derivative.

Remarks:

The proof of the sufficiency of the existence of an ISS Lyanpunov function in Sontag and Wang (1995) constructs the comparison functions $\beta$ and $\gamma$ from $V$ and its estimates. This is important since $\beta$ and $\gamma$ can be used quantitatively. We will not do this for our examples, but want to point out the reference.

We mention, but do not present, further possibilities to define ISS, for instance the concept of "robust stability." An excellent overview can be found in Sontag (2008).

Definitions where the input signal $\mathbf{u}$ and the remaining part of the system depend on time

The ISS definitions presented so far successfully capture the influence of a time-dependent driving signal on an otherwise time-independent system. From this viewpoint, the choice of $\mathbf{u}(t)$ in Eq. 7 is limited to functions that capture all time dependencies. This is a severe limitation if we want to choose a "driving signal" with a particular ecological meaning. An example of this situation that will be discussed 
later is a soil organic matter system driven by the input signal $\mathbf{u}_{O M}(t)$ of the mass of organic matter input, but also by functions dependent on weather conditions that modify decomposition rates such as temperature $\mathbf{u}_{T}(t)$ or moisture $\mathbf{u}_{M}(t)$. If we want to separate the influence of one of these drivers, for instance by choosing $\mathbf{u}=\mathbf{u}_{O M}$, the right-hand side of the ODE will depend on time not only through $\mathbf{u}$, but also directly through $\mathbf{h}$. This is expressed by the $t$ in Eq. 1 . The specific form of $\mathbf{h}$ will be a consequence of our choice of the driving signal $\mathbf{u}$. The most general mathematical concept addressing this situation is input to state stability for time-varying systems (tvISS).

Definition 9 (ISS for time-varying systems) A system is ISS with respect to a nonempty, closed zero-invariant set $\mathcal{A}$ if there exist $\beta \in \mathcal{K} \mathcal{L}$ and $\gamma \in \mathcal{K}$ such that for each initial time $t_{0}$, each initial state $\mathbf{x}_{0}$, each input function $\mathbf{u}$, and all $t \geq t_{0}$

$\left|\mathbf{x}\left(t, \mathbf{x}_{0}, t_{0}, \mathbf{u}\right)\right|_{\mathcal{A}} \leq \beta\left(\left|\mathbf{x}_{0}\right|_{\mathcal{A}}, t-t_{0}\right)+\gamma\left(\|\mathbf{u}\|_{t_{0}, \infty}\right)$

holds.

ISS for time-varying systems can again be characterized by "dissipation": a forward complete time-varying system is ISS with respect to $\mathcal{A}$ if and only if it admits an ISS Lyapunov function $V$ with respect to $\mathcal{A}$.

Definition 10 (ISS Lyapunov function) A smooth function $V: \mathbb{R}^{n} \times \mathbb{R} \rightarrow \mathbb{R}_{\geq 0}$ is an ISS Lyapunov function for system (1) with respect to $\mathcal{A}$ if there exist $\mathcal{K}_{\infty}$ functions $\underline{\alpha}, \bar{\alpha}, \chi$, and a continuous positive definite function $\alpha$ such that

$\underline{\alpha}\left(|\mathbf{x}|_{\mathcal{A}}\right) \leq V(t, \mathbf{x}) \leq \bar{\alpha}\left(|\mathbf{x}|_{\mathcal{A}}\right)$,

and

$|\mathbf{x}|_{\mathcal{A}} \geq \chi(|\mathbf{u}|) \Longrightarrow \frac{\partial V}{\partial t}(t, \mathbf{x})+\nabla V(t, \mathbf{x}) \cdot \mathbf{h}(t, \mathbf{x}, \mathbf{u}) \leq-\alpha\left(|\mathbf{x}|_{\mathcal{A}}\right)$.

Theorem 3 (Edwards et al. (2000) Theorem 1) A forward complete time-varying system (1) is ISS with respect to $\mathcal{A}$ if and only if it admits a smooth ISS Lyapunov function $V$ with respect to $\mathcal{A}$. In the case when $\mathcal{A}$ is compact, the completeness assumption is redundant.

These definitions serve different purposes in the rest of the paper. While the "non-linear superposition" (Theorem 1) and the Lyapunov characterization (Definition 2) are mainly intended to show theoretical connections, the time-varying Lyapunov characterization (Definition 10) will be needed in our most general example application. Definition 5, which is based on a comparison function, and its time-dependent variant (Definition 9) will be useful for the connection to linear time invariant and linear time variant systems, respectively.

\section{Reservoir models}

For the purpose of understanding the examples in this paper, it suffices to interpret all state variables as contents of reservoirs. The interpretation easiest to imagine is content measured in units of mass stored in a reservoir defined by its spatial boundaries. However, reservoirs as well as contents can be much more abstract. The concrete example that motivated the present work is the class of general soil organic matter cycling models described in Sierra and Müller (2015), where the reservoirs are defined by their rates of decomposition rather than their spatial boundaries. It generalizes linear as well as non-linear soil models. Linear examples are as follows: Henin and Dupuis (1945), Henin et al. (1959), Andren and Kätterer (1997), Coleman and Jenkinson (1999), Parton et al. (1987), and Fontaine and Barot (2005). Nonlinear models include the following: Schimel and Weintraub (2003), Sinsabaugh and Follstad Shah (2012), Allison et al. (2010), Zelenev et al. (2000), Wang et al. (2013), and Manzoni and Porporato (2007). Reservoir models also generalize models of the global carbon cycle (Luo and Weng 2011; Rasmussen et al. 2016) and are also commonly used in systems biology (Anderson 2013). It is therefore useful to discuss ISS with respect to this general class of models.

It is also worth mentioning that the ISS theory presented here is in no way limited to models where all state variables describe contents of pools. In particular, ISS permits to discuss systems derived from higher order ODEs, where some of the state variables could still describe reservoir contents. We give a detailed discussion about reservoir models and the property of mass balance in Appendix 2 .

\section{Applications}

\section{A non-linear time-varying soil organic matter decomposition model driven by mass influx}

We start with a prototypical example that shows typical properties of an ISS system with a minimum of technical complexity. It is a place holder for ecologically relevant non-linear systems (Schimel and Weintraub 2003; Sinsabaugh and Follstad Shah 2012; Allison et al. 2010; Zelenev et al. 2000; Wang et al. 2013; Manzoni and Porporato 2007) to be analyzed in the future. Consider the uncoupled system

$\dot{C}_{1}=I_{1}(t)-\left(C_{1}^{2}+C_{1}\right) k_{1}(t)$,
$\dot{C}_{2}=I_{2}(t)-\left(C_{2}^{2}+C_{2}\right) k_{2}(t)$, 
where $C_{1}$ and $C_{2}$ are the carbon contents of two unconnected pools, and the bounded periodic functions $k_{1}$ and $k_{2}$ with

$k_{1}^{\min } \leq k_{1}(t) \leq k_{1}^{\max }$,

$k_{2}^{\min } \leq k_{2}(t) \leq k_{2}^{\max }$

describe the seasonal changes in decomposition speed. One possible choice is

$$
\begin{aligned}
& k_{1}=\frac{k_{1}^{\max }}{2}+\frac{k_{1}^{\text {min }}}{2}+\frac{1}{2}\left(k_{1}^{\text {max }}-k_{1}^{\min }\right) \sin (4 t), \\
& k_{2}=\frac{k_{2}^{\text {max }}}{2}+\frac{k_{2}^{\text {min }}}{2}+\frac{1}{2}\left(k_{2}^{\text {max }}-k_{2}^{\text {min }}\right) \sin (4 t) .
\end{aligned}
$$

If the quadratic terms in $C_{i}^{2}+C_{i}$ were missing, the model would be linear and the $k_{i}$ would be called decomposition rates (Olson 1963). $I_{1}$ and $I_{2}$ are mass fluxes to the system. The system can have a fixed point

$\overline{\mathbf{C}}=\left(\begin{array}{l}\bar{C}_{1} \\ \bar{C}_{2}\end{array}\right)$,

if the input streams have the same period and phase as the decomposition rates:

$\mathbf{I}^{0}(t)=\left(\begin{array}{l}\left({\overline{C_{1}}}^{2}+\bar{C}_{1}\right) k_{1}(t) \\ \left({\overline{C_{2}}}^{2}+\bar{C}_{2}\right) k_{2}(t)\end{array}\right)$.

The invariant set of our definitions reduces to one point:

$\mathcal{A}=\{\overline{\mathbf{C}}\}$.

We will now disturb both mass influxes individually by perturbations $u_{1}(t)$ and $u_{2}(t)$ and get

$\dot{C}_{1}=I_{1}^{0}(t)+u_{1}(t)-\left(C_{1}^{2}+C_{1}\right) k_{1}(t)$,

$\dot{C}_{2}=I_{2}^{0}(t)+u_{2}(t)-\left(C_{2}^{2}+C_{2}\right) k_{2}(t)$.

Note that the equivalence $\mathbf{I}=\mathbf{I}^{0}+\mathbf{u}$ should be read from right to left: $\mathbf{u}=\mathbf{I}-\mathbf{I}^{0}$. For an arbitrary given $\mathbf{I}(t)$, the choice of $\mathbf{I}^{0}(t)$ implicitly defines $\mathbf{u}(t)$. As a consequence, even the most ideal choice of a reference is not artificial at all. It just defines what we call a disturbance. Inside the ISS framework, it is enough to know its size $\|\mathbf{u}\|_{t_{0}, \infty}$. For instance, imagine that the fluxes are given as time series data. By subtracting a reference $\mathbf{I}^{0}(t)$, one gets a time series for the perturbations $\mathbf{u}(t)$ with respect to the reference. $\|\mathbf{u}\|_{t_{0}, \infty}$ is just the biggest value of the $|\mathbf{u}|$ time series. We could also choose a constant reference input:

$\mathbf{I}^{0}=$ const. $=\left(\begin{array}{l}z_{1} \\ z_{2}\end{array}\right)$.
The system then no longer has a fixed point for $\mathbf{u}=\mathbf{0}$, but a rectangular invariant set

$\mathcal{A}=\left\{\left(C_{1}, C_{2}\right): \begin{array}{l}\bar{C}_{1}^{\min } \leq C_{1}(t) \leq \bar{C}_{1}^{\max } \\ \bar{C}_{2}^{\text {min }} \leq C_{2}(t) \leq \bar{C}_{2}^{\max }\end{array}\right\}$

where $\bar{C}_{1}^{\min }$ and $\bar{C}_{1}^{\max }$ are the fixed points of the two minimum and maximum $k_{1}$ rate systems:

$0=-k_{1}^{\min }\left(\bar{C}_{1}^{\min ^{2}}+\bar{C}_{1}^{\text {min }}\right)+z_{1}$,

$0=-k_{1}^{\max }\left(\bar{C}_{1}^{\max ^{2}}+\bar{C}_{1}^{\max }\right)+z_{1}$

and $\bar{C}_{2}^{\min }$ and $\bar{C}_{2}^{\max }$ are the fixed points of the two respective minimum and maximum $k_{2}$ rate systems:

$0=-k_{2}^{\min }\left(\bar{C}_{2}^{\min ^{2}}+\bar{C}_{2}^{\min }\right)+z_{2}$,

$0=-k_{2}^{\max }\left(\bar{C}_{2}^{\max }{ }^{2}+\bar{C}_{2}^{\max }\right)+z_{2}$.

As we will prove later, both versions of the system exhibit the ISS property. We can draw a number of interesting conclusions from this system (Fig. 2).

1. The four plots at the top show the disturbances.

(a) The upper left shows the absolute value of two different disturbances in different colors as functions of time. The graphs fit into semi-transparent rectangles. The height of the rectangles is given by the infinity norm of the absolute value of the disturbance and is the only relevant information that the ISS statements will refer to.

(b) The lower left and upper right plots show the arbitrarily chosen components of $\mathbf{u}$ given by functions of the form $u_{i}(t)=A_{i} \sin \left(\omega_{i} t\right)$. The height of the $u_{2}$ rectangle, and the width of the $u_{1}$ rectangle are given by the infinity norm of the absolute value of $\mathbf{u}$, which is not the smallest possible upper bound, as the picture shows, since the graphs do not fill the whole height or width the rectangle would allow.

(c) The lower right plot shows how an upper bound of the infinity norm confines trajectories of disturbances in the $\vec{u}$-space to disks. The $y$-axis is shared with the plot for $u_{2}$ to the left and the $x$-axis with the plot for $u_{1}$ above. Looking at the confined components, suggestively plotted to the left and on top, one expects the regions for the disturbances in the $\left(u_{1}, u_{2}\right)$-plane to be rectangles. While this expectation is correct, it is not used in the ISS definition, which refers to the infinity norm of the vector $\mathbf{u}$.

2. The next four plots in the middle show the effect of these disturbances on the solutions for the system with a fixed point.

(a) The upper left plot shows the absolute value of the deviations of solutions from the fixed point as 

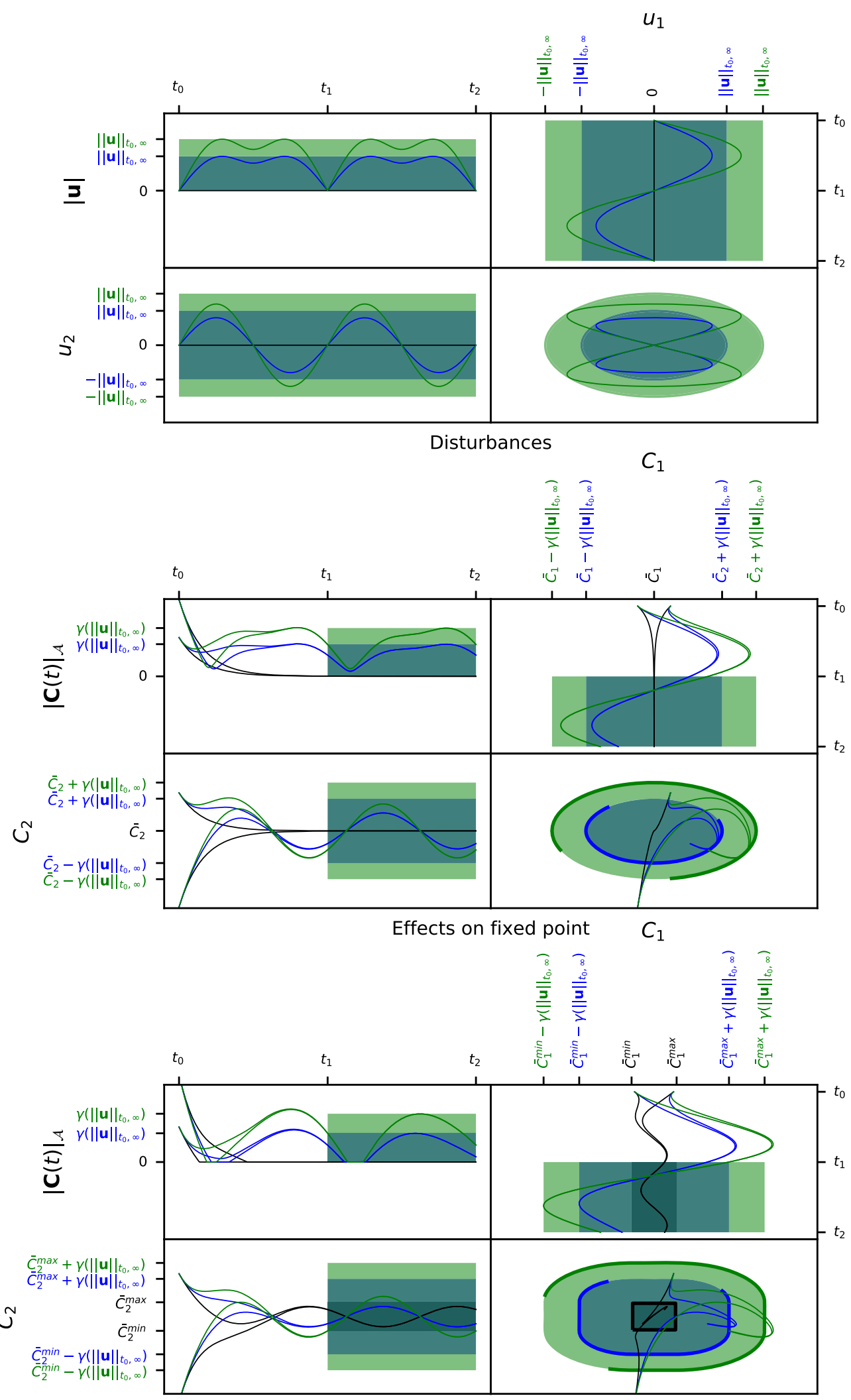

Effects on an invariant rectangle

Fig. 2 Effects of disturbances with respect to different $\mathbf{I}^{0}$. The upper panels show the disturbances, the middle panels show the effect of the disturbance on the system with a fixed point, and the lower panels show the effect of the disturbances on the system that has no fixed points. See the main text for a detailed explanation of the plots 
functions of time. We choose two different start positions $\left(C_{1}, C_{2}\right)$ with different distances to the fixed point $\left(\bar{C}_{1}, \bar{C}_{2}\right)$. For every start position, we compute the undisturbed solution, represented by the black graph, and one for each of the two disturbances represented by the same color as the disturbance influencing it. At the beginning, the influence of the start position is clearly discernible, but as time goes on, this influence decreases, and we can predict the distance of the solution to the fixed point to be bounded by a similar rectangle. For an ISS system, the height of this rectangle is a monotone function $\gamma$ of $\|\mathbf{u}\|_{t_{0}, \infty}$ with $\gamma(0)=0$. We can formulate both statements in the reservoir language of our example: A limited disturbance in the mass fluxes will eventually lead to a limited aberration of the pool contents from the equilibrium value. We can predict the (eventual) aberration of the pool sizes to be smaller for the smaller disturbance. The fact that $\gamma(0)=0$ means that our system will converge to its fix point if we do not disturb it.

(b) The lower left and upper right plots show the effect of the disturbances on the components of $\mathbf{C}$. Since $|\mathbf{C}|$ has to approach a limited region, so too must its components. We also see again that a tight bound on $|\mathbf{C}|$ is not necessarily tight on the components.

(c) The lower right plot shows how an upper bound of the norm confines trajectories in the phase space to disks. The $y$-axis is shared with the plot for $C_{2}$ to the left and the $x$-axis with the plot for $C_{1}$ above. Looking at the confined components, one again expects the regions to be rectangles, but since ISS does not make any statements about the components, but about the norm of the whole vector, the rectangles we could construct would again only circumscribe the disks, thereby weakening the statement. Translated into reservoir terms, we can expect the reservoir contents with the blue disturbance of the influx to end up in the blue region of the state space. For the bigger green disturbance, we can expect the solution eventually somewhere in the bigger green disk.

3. The four plots at the bottom show the effect of these disturbances on the solutions for the system, which no longer has a fixed point, but at least a zero-invariant set.

(a) The upper left plot shows the absolute value of the deviations of solutions from the invariant set as functions of time. The plot states the same result as its counterpart for the fixed point. The only difference is that the distance to a point is replaced by the distance to a set. This distance is what ISS is concerned with. Eventually, all solutions will end up in the vicinity of the invariant set regardless of the start value. The smaller the disturbance is, the closer to the set they will be.

(b) The lower left and upper right plots show the effect of the disturbances on the components of $\mathbf{C}$. Let us first look at the undisturbed (black) solutions only. In our example, the combination of constant mass influxes to both reservoirs and a time dependent decomposition leads to changing pool contents. The plot suggests that the contents of the reservoirs follow the oscillations of the decomposition, and more importantly, they vary only in a certain range. For this simple example, it is straightforward to show that these ranges can be established. We can show that once an unperturbed solution enters the region defined by the ranges, it will never leave it. The region is invariant. The ISS property does not tell us anything about this region, but about the distances of solutions to it. The big green disturbance leads to a big boundary layer around the invariant set. The smaller blue disturbance leads to solutions ending up in the smaller blue boundary layer. All solutions, regardless of their start values, will eventually end up there.

(c) The lower right plot shows how an upper bound on the norm of the distance confines trajectories in the phase space. The invariant set is a rectangle due to the fact that in our simple example, the reservoir contents are not coupled. In general, the invariant set can have a different shape; the ISS property just ensures that we will eventually find solutions in an area defined by a distance to the set depending monotonically on the size of the disturbance.

Up to now, we have only argued that our system looks like an ISS system since the expected properties show up in the plots. Of course, we have to prove this. The idea of the proof is suggested by the result of Theorem 3: We have to find an ISS Lyapunov function in the sense of Definition 10. We first note that the positive orthant is an invariant set for the system defined by Eqs. 10 and 11 since it is a reservoir system and so derivatives point inward at the boundaries (Jacquez and Simon 1993). That $\mathcal{A}=\left[\bar{C}_{1}^{\text {min }}, \bar{C}_{1}^{\text {max }}\right] \times$ $\left[\bar{C}_{2}^{\text {min }}, \bar{C}_{2}^{\text {max }}\right]$ is zero invariant does not have to be proved separately if we find an ISS Lyapunov function for this set. We propose $V=\frac{1}{2}|\mathbf{C}|_{\mathcal{A}}^{2}$ as such a Lyapunov candidate. We do not need to make it explicitly time dependent although this would provide additional freedom in some cases. Note first that $V$ is smooth, positive definite, and we can easily choose equal lower and upper limiting functions

$\underline{\alpha}\left(|\mathbf{C}|_{\mathcal{A}}\right)=\bar{\alpha}\left(|\mathbf{C}|_{\mathcal{A}}\right)=V(\mathbf{C})$ 
We now show that we can find $\alpha, \chi \in \mathcal{K}^{\infty}$ such that for $|\mathbf{C}|_{\mathcal{A}} \geq \chi(|\mathbf{u}|$ ) (inputs small enough in comparison to the distance), the orbital derivative can be estimated by $\dot{V} \leq$ $-\alpha\left(|\mathbf{C}|_{\mathcal{A}}\right)$.
The piecewise definition of $V$ leads to the piecewisedefined derivative:

$$
\begin{aligned}
\dot{V}= & |\mathbf{C}|_{\mathcal{A}} \frac{d}{d t}|\mathbf{C}|_{\mathcal{A}} \\
= & \begin{cases}0 & : \bar{C}_{i}^{\text {min }} \leq C_{i} \leq \bar{C}_{i}^{\text {max }} i \in\{1,2\} \\
\left(\bar{C}_{1}^{\text {min }}-C_{1}\right) \dot{C}_{1}+\left(C_{2}-\bar{C}_{2}^{\text {max }}\right) \dot{C}_{2} & : C_{1}<\bar{C}_{1}^{\text {min }} \text { and } C_{2}>\bar{C}_{2}^{\text {max }} \\
\left(\bar{C}_{2}^{\text {max }}-C_{2}\right) \dot{C}_{2} & : \bar{C}_{1}^{\text {min }} \leq C_{1} \leq \bar{C}_{1}^{\text {max }} \text { and } C_{2}>\bar{C}_{2}^{\text {max }} \\
\left(C_{1}-\bar{C}_{1}^{\text {max }}\right) \dot{C}_{1}+\left(C_{2}-\bar{C}_{2}^{\text {max }}\right) \dot{C}_{2} & : C_{1}>\bar{C}_{1}^{\text {max }} \text { and } C_{2}>\bar{C}_{1}^{\text {max }} \\
\left(C_{1}-\bar{C}_{1}^{\text {max }}\right) \dot{C}_{1} & : C_{1}>\bar{C}_{1}^{\text {max }} \text { and } \bar{C}_{2}^{\text {min }}<C_{2}<\bar{C}_{2}^{\text {max }} \\
\left(C_{1}-\bar{C}_{1}^{\text {max }}\right) \dot{C}_{1}-\left(\bar{C}_{2}^{\text {min }}-C_{2}\right) \dot{C}_{2} & : C_{1}>\bar{C}_{1}^{\text {max }} \text { and } C_{2}<\bar{C}_{2}^{\text {min }} \\
-\left(\bar{C}_{2}^{\text {min }}-C_{2}\right) \dot{C}_{2} & : C_{2}<\bar{C}_{2}^{\text {min }} \text { and } \bar{C}_{1}^{\text {min }}<C_{1}<\bar{C}_{1}^{\text {max }} \\
-\left(\bar{C}_{1}^{\text {min }}-C_{1}\right) \dot{C}_{1}-\left(\bar{C}_{2}^{\text {min }}-C_{2}\right) \dot{C}_{2} & : C_{1}<\bar{C}_{1}^{\text {min }} \text { and } C_{2}<\bar{C}_{2}^{\text {min }} \\
-\left(\bar{C}_{1}^{\text {min }}-C_{1}\right) \dot{C}_{1} & : C_{1}<\bar{C}_{1}^{\text {min }} \text { and } \bar{C}_{2}^{\text {min }}<C_{2}<\bar{C}_{2}^{\text {max }}\end{cases}
\end{aligned}
$$

Since our system is uncoupled, the following estimates can be derived by combining estimates from scalar systems of the form

$$
V_{i}=\left|C_{i}\right|_{\mathcal{A}}^{2}= \begin{cases}0 & : \bar{C}^{\text {min }} \leq C_{i} \leq \bar{C}^{\text {max }} \\ \left(C_{i}-\bar{C}_{i}^{\text {min }}\right)^{2}: C_{i}<\bar{C}_{i}^{\text {min }} \\ \left(C_{i}-\bar{C}_{i}^{\text {max }}\right)^{2}: C_{i}>\bar{C}_{i}^{\text {max }} .\end{cases}
$$

with $V\left(|\mathbf{C}|_{\mathcal{A}}\right)=V_{1}\left(\left|C_{1}\right|_{\mathcal{A}}\right)+V_{2}\left(\left|C_{2}\right|_{\mathcal{A}}\right)$. In particular, we have

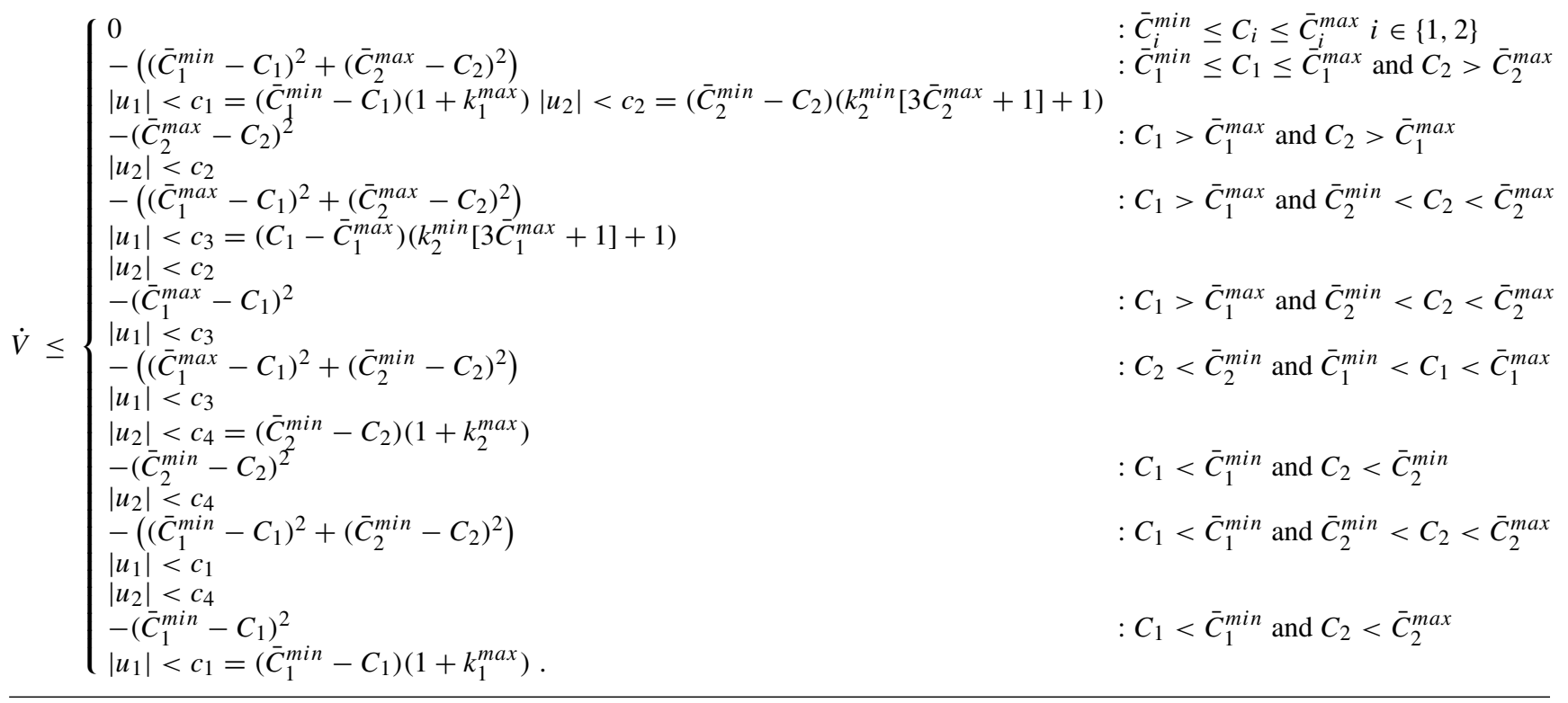

We can choose $\mu(|\mathbf{u}|)=\frac{|\mathbf{u}|}{c}$ with $c=\max \left\{c_{1}, \cdots, c_{4}\right\}$ and $\alpha\left(|\mathbf{C}|_{\mathcal{A}}\right)=|\mathbf{C}|_{\mathcal{A}}^{2}$. The proof for the fixed point is implicitly included. In this case, $\bar{C}_{i}^{\min }=\bar{C}_{i}^{\max }=\bar{C}_{i}$ and $k_{i}^{\text {min }}=k_{i}^{\max }$.

Remarks:

It is interesting to ask what we could learn about the examples by classical stability theory for autonomous systems. The first thing we would have to do is to get rid of the time dependency. But it turns out that the rate of change of the state variables in a quasi-static approximation is comparable to the rate of change of the driving signal and the rate of change of the parameters in the compartmental matrix. Consequently, a quasi-static approximation would be misleading and is therefore not an option. 
Another thing one could try is to consider averaging with respect to time. Incidentally, the example encourages this through the periodicity of the driving signal and the parameter changes. Of course, this would not help to find out how much the state variables, the contents of the pools, change with time. To get an idea about the amplitude of such changes, one could try to choose minimal and maximal values for input and decomposition rates and observe the behavior of the resulting systems in the phase plane. For the examples and their extremely regular and periodic parameter changes, the results would not be too misleading. This is, however, not always the case as the simple example in the introduction showed. Resonance effects cannot be taken into account in the autonomous view, but could lead to diverging solutions.

Another question is how one would approach the problem from a non-autonomous perspective. Here the stability of a trajectory is a more natural concept than the stability of a point (Kloeden and Rasmussen 2011). In the compartmental model of the example, we do not expect a fixed point but periodic solutions for periodic inputs, caused for instance by seasonal cycles. We could now interpret the input as a control and the whole system following this control. Instead of treating the whole seasonal variation as deviation, we could consider the deviation from the expected solution under the influence of a given uncertainty in the driving input. An ISS system will stay close to the expected solution. ISS was originally developed in control theory to guarantee that a feedback designed to stabilize a system still does so if the feedback is not completely correct, but exhibits a bounded uncertainty. ISS for time-varying systems has been applied to tracking problems (Tsinias and Karafyllis 1999).

\section{A non-linear influx controlled compartmental model that is not ISS}

It is important to see that not all compartmental models are ISS. For instance, we give the following example describing an enzyme-controlled degradation of some substrate $S$ according to Michaelis-Menten kinetics:

$\dot{S}=I-V^{\max } \frac{S}{K_{M}+S}$,

where $V^{\max }$ is the maximum rate of the reaction and the Michaelis constant $K_{M}$ is the substrate concentration at which the reaction rate is $\frac{1}{2} V^{\max }$. For a constant $I^{0}<$ $V^{\max }$, the equation has a fixed point

$\bar{S}=\frac{I K_{M}}{V^{\max }-I^{0}}$.

It is easy to see that this fixed point is GAS in $\mathbb{R}^{+}$, for instance by Jacquez and Simon (1993) Theorem 5, which covers compartmental systems whose Jacobian is itself a compartmental matrix (Definition 18 in Appendix 2). In this simple one-dimensional case, the Jacobian is the derivative $\frac{d \dot{S}}{d S}=-\frac{K V^{\max }}{(K+S)^{2}}$, which is itself a one-dimensional "compartmental matrix," but it is clear that the input-controlled system

$\dot{S}=u(t)+I^{0}-V^{\max } \frac{S}{K_{M}+S}$

cannot be ISS, since for any constant $u^{0}>V^{\max }-I^{0}, S$ would increase beyond all bounds.

Remarks:

If additional knowledge of the bounds of $\|u\|_{t_{0}, \infty}$ is available (in this example $\|u\|_{t_{0}, \infty} \leq \alpha=\min \left\{V^{\max }-I^{0}, I^{0}\right\}$ ), one could make the ISS definition more specific by a condition on the size of $\|u\|_{t_{0}, \infty}$. For practical purposes, it might be enough to show that a system is ISS for $\|u\|_{t_{0}, \infty}<\alpha$. For this simple example, this conditional ISS property could be achieved.

In this case, classical bifurcation analysis with respect to the parameter $I^{0}$ representing a constant input shows that the fixed point disappears for $I^{0}>V^{\max }$, which helps to exclude ISS. However, as we have seen in the introduction, classical methods are not sufficient to establish ISS. Note that ISS is a form of stability denoted as bounded input bounded states (BIBS).

Definition 11 (BIBS stability) System (1) is BIBS if bounded inputs lead to bounded states:

$\|\mathbf{u}\|_{t_{0}, \infty}<\delta \Longrightarrow\|\mathbf{x}\|_{t_{0}, \infty}<\varepsilon$

For autonomous compartmental systems, a necessary and sufficient condition for BIBS is the following (Bastin 1999): Let $q_{i}(\mathbf{x})$ denote the output flux from pool $i$ and $M=\sum_{i} x_{i}$ the overall mass of the system. Assume that the input $\mathbf{I}(t)$ is bounded $0 \leq I_{i}(t) \leq I_{i}^{\max }$ for $t \geq t_{0} i \in 1, \ldots, n$. Assume further that the cumulative outflux $\sum_{i} q_{i}$ depends on the cumulative mass $M$ in such a way that for any $I^{\max }$, we can find a mass $M^{0}$ such that $M(\mathbf{x})>M^{0} \Longrightarrow$ $\sum_{i} q_{i}(\mathbf{x})>I^{\max }$. Then the state $\mathbf{x}$ is bounded and the simplex $\Delta=\left\{\mathbf{x} \in \mathbb{R}^{+^{n}}: M(\mathbf{x}) \leq M^{0}\right\}$ is an invariant set.

In our case, where $M=x=S$, and $\lim _{S \rightarrow \infty} q(S)=$ $V^{\max }$, this condition is clearly violated and the output flux cannot compensate inputs $I>V^{\max }$ even if $S \rightarrow \infty$. Of course, such systems cannot be ISS.

While this criterion might be very useful to exclude the possibility of ISS, it also raises the question what the simplest system that can compensate arbitrary inputs would look like. To see this, let us temporarily adopt the mind-set of control theory and consider the task to stabilize an inputcontrolled compartmental system at a prescribed fixed point $\bar{x}$. For any fixed $I$, we look for a control law $u(x)$ such that the influx is compensated. The most intuitive approach is to make the control proportional and opposite in direction to 
the misfit of the state, $u=-k(x-\bar{x})$ with $k>0$. We write the equilibrium condition in the following way:

$$
\begin{aligned}
\dot{x} & =0 \\
& =I+u(\bar{x}) \\
& =I-k(x-\bar{x}) \\
& =\tilde{I}-k(x) .
\end{aligned}
$$

The resulting system is linear, with $\tilde{I}=I-k \bar{x}$. If we read the last equations backward, they say that a linear system can "control" its inputs with respect to its fixed point, by scaling countermeasures according to the misfit. It is easy to see that such systems fulfill the abovementioned condition for BIBS and are therefore good candidates for ISS and a motivation for the next subsection. Linear systems will serve as a prototype that guides the search for ISS systems. On the other hand, the example reveals an important implicit assumption of linear models, namely the ability to increase the size of the counteracting signal to any size demanded by the influx. A control engineer can guarantee such an idealized controller usually only in a specific interval of operation. It is certainly wise to keep this in mind for natural systems too.

\section{Linear models}

\section{Linear time variant models}

Many reservoir models (Coleman and Jenkinson 1999; Anderson 2013; Xia et al. 2013; Raupach 2013; Sierra and Müller 2015) can be written in the general form:

$\dot{\mathbf{C}}=\mathbf{f}(t, \mathbf{C}, \mathbf{I}(t))=\mathbf{A}(t) \mathbf{C}+\mathbf{I}(t)$

To establish the connection to the last example and the nomenclature of control theory, we choose a fixed point $\overline{\mathbf{C}}$, a fitting $\mathbf{I}_{0}(t)=-\mathbf{A}(t) \overline{\mathbf{C}}$, and a new variable $\mathbf{x}=\mathbf{C}-\overline{\mathbf{C}}$, so that our system now reads

$$
\begin{aligned}
\dot{\mathbf{x}} & =\mathbf{A}(t) \mathbf{x}+\mathbf{A}(t) \overline{\mathbf{C}}+\mathbf{I}_{0}(t)+\mathbf{u}(t) \\
& =\mathbf{A}(t) \mathbf{x}+\mathbf{u}(t) .
\end{aligned}
$$

The fixed point is now $\mathbf{x}=\mathbf{0}$. In control theory, linear models are usually written a little bit more general by allowing a linear transformation of the signal:

$\dot{\mathbf{x}}(t)=\mathbf{A}(t) \mathbf{x}(t)+\mathbf{B}(t) \mathbf{u}(t)$.

Although for the influx-controlled systems we have been looking at so far $\mathbf{B}=\mathbf{1}$, we will adopt the more general notation here to allow the reader to use another input signal. One can write the solution of Eq. 17 in the form

$\mathbf{x}(t)=\Phi\left(t, t_{0}\right) \mathbf{x}_{0}+\int_{t_{0}}^{t} \Phi(t, \tau) \mathbf{B}(\tau) \mathbf{u}(\tau) d \tau$, where $\Phi$, defined by $\Phi\left(t, t_{0}\right) \mathbf{x}\left(t_{0}\right)=\mathbf{x}(t)$, is called the state transition matrix. Most interestingly, $\Phi$ can be derived from the input-free system

$\dot{\mathbf{x}}(t)=\mathbf{A}(t) \mathbf{x}(t)$,

and is therefore independent of $\mathbf{u}$. We prove the following simple theorem that connects linear control to ISS. Its importance does not consist in its mathematical depth, but in the connection it establishes to a large class of models that we want to study.

Let $\|\cdot\|$ denote some matrix norm, for instance the Frobenius norm, induced by the Euclidean vector norm. (Due to the equivalence of norms on $\mathbb{R}^{n}$, any pair of vector norm and induced matrix norm is possible.)

Theorem 4 (sufficient condition for ISS for linear timevarying systems)

The linear system (17) is ISS if it is uniformly asymptotically stable and $\mathbf{B}(t)$ is bounded, i.e.,

$\exists \eta, \lambda>0:\left\|\Phi\left(t, t_{0}\right)\right\| \leq \eta e^{-\lambda\left[t-t_{0}\right]}$, for all $t \geq t_{0}$

$\exists k_{B}>0:\|\mathbf{B}(t)\| \leq k_{B}$, for all $t$.

Proof We have to show that we can find $\beta \in \mathcal{K} \mathcal{L}$ and $\gamma \in$ $\mathcal{K}$ :

$$
\begin{aligned}
|\mathbf{x}(t)| & =\left|\Phi\left(t, t_{0}\right) \mathbf{x}_{\mathbf{0}}+\int_{t_{0}}^{t} \Phi\left(\tau, t_{0}\right) \mathbf{B}(\tau) \mathbf{u}(\tau) d \tau\right| \\
& \leq \eta e^{-\lambda\left[t-t_{0}\right]}\left|\mathbf{x}_{0}\right|+\int_{t_{0}}^{t} \eta e^{-\lambda[t-\tau]} k_{B}\|\mathbf{u}\|_{t_{0}, \infty} d \tau \\
& =\eta e^{-\lambda\left[t-t_{0}\right]}\left|\mathbf{x}_{0}\right|+\frac{\eta k_{B}\|\mathbf{u}\|_{t_{0}, \infty}}{\lambda}\left[1-e^{-\lambda\left(t-t_{0}\right)}\right] \\
& \leq \underbrace{\eta e^{-\lambda\left[t-t_{0}\right]}\left|\mathbf{x}_{0}\right|}_{\beta\left(\left|\mathbf{x}_{0}\right|, t\right)}+\underbrace{\frac{\eta k_{B}\|\mathbf{u}\|_{t_{0}, \infty}}{\lambda}}_{\leq \gamma\left(\|u\|_{t 0, \infty}\right)} .
\end{aligned}
$$

Remarks:

Up to now, we have referred to the comparison functions $\beta$ and $\gamma$ in a qualitative way only. The very explicit form they take in the last line of the proof presents the opportunity to point out that they are very useful in a quantitative way too: given the start value and the size of the input they confine solutions at any given time.

Uniform asymptotic stability is stronger than ISS. It follows that if we were concerned with linear systems only, we would not need ISS. We present it here since ISS extends familiar notions of stability to the non-linear case. Considering a given non-linear system, it can also be very useful to find a linear system with a weaker stabilizing feedback that is already sufficient for stability. We will use this reasoning for one of our examples. 
In applications, it is easy to check Eq. 20. In the case of influx-controlled systems, $\mathbf{B}(t)=\mathbf{1}$.

A nice (but not necessary) starting position to prove Eq. 19 would be a closed expression for $\Phi\left(t, t_{0}\right)$. This is unfortunately not available in general, but some very useful special cases are related to properties of compartmental systems:

1. If $\mathbf{A}$ is diagonal, so is $\Phi$ :

$$
\begin{aligned}
& \mathbf{A}(t)=\left\{\begin{array}{llll}
a_{1}(t) & 0 & \cdots & 0 \\
0 & \ddots & \ddots & \vdots \\
\vdots & \ddots & \ddots & 0 \\
0 & \ldots & 0 & a_{n}(t)
\end{array}\right\}
\end{aligned}
$$

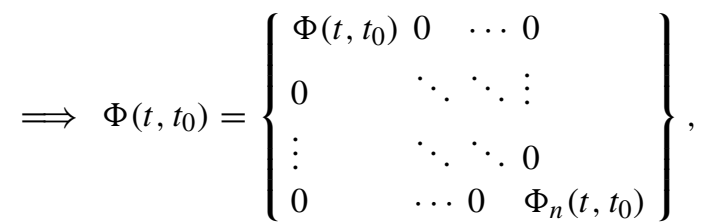

with

$\Phi_{i}\left(t, t_{0}\right)=e^{\int_{t_{0}}^{t} a_{i}(\tau) d \tau}$.

So for parallel linear models, where all the reservoirs of the model have only connections to the environment via inputs and outputs, but not to other reservoirs, Eq. 19 can be checked component-wise for the elements (22), which is a huge simplification. If, for example, one has $\min _{t>t_{0}} a(t)<-b<0$, Eq. 19 holds.

2. If $\mathbf{A}$ is triangular so is $\Phi$ :

$$
\begin{aligned}
\mathbf{A}(t) & =\left\{\begin{array}{llll}
a_{1,1}(t) & 0 & \cdots & 0 \\
\vdots & \ddots & \ddots & \vdots \\
\vdots & \ddots & 0 \\
a_{n, 1}(t) & \cdots & \cdots & a_{n, n}(t)
\end{array}\right\} \Longrightarrow \\
\Phi\left(t, t_{0}\right) & =\left\{\begin{array}{llll}
\Phi_{1,1}\left(t, t_{0}\right) & 0 & \cdots & 0 \\
\vdots & & \ddots & \ddots \\
\vdots & & \ddots & \vdots \\
\Phi_{n, 1}\left(t, t_{0}\right) & \cdots & \cdots & \Phi_{n, n}\left(t, t_{0}\right)
\end{array}\right\},
\end{aligned}
$$

with $\Phi_{i, i}\left(t, t_{0}\right)=e^{\int_{t_{0}}^{t} a_{i, i}(\tau) d \tau}$ and $\Phi_{i, j}\left(t, t_{0}\right)$ for $j \neq i$ by back-substitution into the ODE. The triangular structure of $\mathbf{A}$ reflects an ordering of reservoirs where content moves only in the direction of pools with increasing indices. Such an ordering can be achieved, for example, if it is known that energy or mass flows only in one direction, leading to a special (linear) cascade (see "Cascades of ISS systems" section).

3. If there exists some constant matrix $\mathbf{M}$ such that $\mathbf{A}=$ $a(t) \mathbf{M}$, then $\Phi$ is given as the matrix exponential

$\Phi\left(t, t_{0}\right)=e^{\left[\int_{t_{0}}^{t} a(\tau) d \tau \mathbf{M}\right]}$.
In the special cases mentioned, Eq. 19 can be established by looking at the concrete components $a_{i, j}(t)$ of the model. The $a_{i, j}(t)$ may be under restriction by ecological principles and an upper bound might be derived from the expression for $\Phi$. Even without the knowledge of $\Phi$, ecologically implied properties of $\mathbf{A}$ can be successfully exploited. A very interesting example has recently been proved in Rasmussen et al. (2016), which requires the following definition to state it.

Definition 12 (Strict diagonal dominance) The $m \times m$ matrix $\mathbf{B}(t)$ is called strictly diagonally dominant if there exists a $\delta>0$ such that

1. $\quad \mathbf{B}(t)_{i, i}<0$ for all $t$ and $i \in\{1, \ldots m\}$

2. $\quad \mathbf{B}(t)_{i, j} \geq 0$ for all $t$ and $i \neq j \in\{1, \ldots m\}$

3. $\sum_{j=1}^{m} \mathbf{B}(t)_{i, j} \leq-\delta$ for all $t$ and $i \in\{1, \ldots m\}$

If $\mathbf{A}$ is a lower triangular block matrix of strictly diagonally dominant blocks (including the case that $\mathbf{A}$ is the only block), then there exist constants $K \geq 1$ and $\gamma>0$ such that

$$
\left\|\Phi\left(t, t_{0}\right)\right\| \leq K e^{-\gamma\left(t-t_{0}\right)} .
$$

This result generalizes an older one for linear timedependent, donor-controlled systems (where the outflows of pool $j$ only depend on $C_{j}$ instead of the whole vector $\mathbf{C}$, which is always the case for linear systems) with strict diagonal dominance (Mulholland and Keener 1974), where the one block version was used to investigate periodic systems. In Appendix 2.2, we show that assuming strict diagonal dominance is justified for soil organic matter (SOM) decomposition models where microbial activity and respiration never stop in any of the pools.

\section{Linear time invariant models}

For LTI models, A and B do not depend on time and the state transition operator is known explicitly as the matrix exponential:

$$
\begin{aligned}
\dot{\mathbf{x}} & =\mathrm{A} \mathbf{x}+\mathrm{Bu}(t) \\
\Phi\left(t, t_{0}\right) & =\mathbf{e}^{\left(t-t_{0}\right) \mathbf{A}} .
\end{aligned}
$$

If we based our discussion on Eq. 18 only, we could state that if $A$ is Hurwitz (all eigenvalues have negative real parts), ISS follows. Since Eq. 18 is sufficient, but in the general time-dependent case not necessary, it is interesting to see if we can weaken it for LTI systems. For fixed points, it is well known that this is not the case and it is also necessary that the real parts of all eigenvalues are smaller than zero. If we were only interested in ISS with respect to equlibria, we could close this section by a reference to the original 
proof and avoid the discussion of zero real part eigenvalues completely. It is however interesting to discover the physical meaning of such eigenvalues for compartmental systems (Definition 17 in Appendix 2). This will enable us to imagine invariant sets larger than equilibria. Furthermore, some textbooks (Braun 1993) (correctly) claim stability of LTI systems for cases of eigenvalues with zero real part. The closer look that we are going to take reveals that the definition of stability used there is not adequate for systems with inputs. Therefore, we discuss reservoir models along two lines.

1. Cases of invariant sets, the ISS property can be established for

(a) A fixed point

(b) A linear subspace of the phase space

2. Cases of the eigenvector structure (represented by the Jordan normal form (JNF)) of A:

(a) All eigenvalues $\left\{\lambda_{i}\right\}$ of $\mathbf{A}$ have negative real part.

(b) A has no eigenvalues with positive real part, but there are eigenvalues with zero real part.

i. The geometric multiplicity of the eigenvalues with zero real part is equal to their algebraic multiplicity.

A. The imaginary part is zero for all eigenvalues where the real part is.

B. There are eigenvalues with zero real part and non-zero imaginary part.

ii. The geometric multiplicity of the eigenvalues with zero real part is smaller than their algebraic multiplicity.

(c) There are eigenvalues with positive real part.

Some cases under 2 are incompatible with compartmental matrices. Hearon (1963) and Hearon (1953) has shown that eigenvalues of compartmental systems have non-positive real parts and cannot be purely imaginary. This excludes $2 \mathrm{c}$ and $2 \mathrm{biB}$. According to the discussion of the proof of Theorem 2 in Jacquez and Simon (1993), the case 2bii is also not possible for LTI compartmental systems, which have as many compartments without outflow (simple traps) as zero eigenvalues with linear independent eigenspaces.

We start our discussion with the combination 1a 2a. Although the result could have been derived from Sontag (1998b), we state and prove it in the ISS context as

Theorem 5 System (23) has the ISS property with respect to a fixed point (of the input-free system) if and only if all eigenvalues $\left\{\lambda_{i}\right\}$ of $\mathbf{A}$ have negative real part.

\section{Remark:}

While we consider $\mathbf{u}=\mathbf{u}(t)$ for ISS, we mean the fixed point resulting from $\mathbf{u}=\mathbf{0}$. Since a variable transformation is always possible, this covers any fixed influx $\mathbf{I}_{0}$.

Proof $\Longrightarrow$ (negative real parts imply ISS)

According to Theorem 4, it is sufficient to show that the system is uniformly asymptotically stable. We have to show

$$
\begin{aligned}
& \exists \lambda, \gamma>0 \text { such that } \\
& \left\|\Phi\left(t, t_{0}\right)\right\|=\left\|\mathbf{e}^{\left(t-t_{0}\right) \mathbf{A}}\right\| \leq \gamma e^{-\lambda\left[t-t_{0}\right]}, \text { for all } t \geq t_{0} .
\end{aligned}
$$

We can choose $\lambda$ as the largest real part: $\lambda=\max \left\{\Re\left(\lambda_{i}\right)\right\}$ and $\gamma=1$.

\section{$\Longleftarrow$ (negative real parts are implied by ISS)}

To show that negative real parts are also necessary for ISS, we first note that the cases under point 2 form a partition. No other cases are possible. We now have to look at the concrete form of $\mathbf{e}^{\left(t-t_{0}\right) \mathbf{A}}$ for each of them. From the discussion above, we know that the cases $2 \mathrm{c}, 2 \mathrm{bii}$, and $2 \mathrm{biB}$ do not occur for compartmental systems. For 2 bi, the system has at least one trap and the input-free system can have (infinitely many) fixed points (namely the initial values of the pools in the traps), but the integral term in Eq. 18 does not generally vanish for $\mathbf{u} \neq \mathbf{0}$. The restriction of $\mathbf{e}^{\left(t-t_{0}\right) \mathbf{A}}$ on the subspace, spanned by the eigenvectors for eigenvalue 0 , becomes the identity $\mathbf{1}$ and even for a constant $\mathbf{u}, \lim _{t \rightarrow \infty} \int_{t_{0}}^{t} \mathbf{1} \mathbf{B} \mathbf{u}(\tau) d \tau$ will not exist even though $\|\mathbf{u}\|_{t_{0}, \infty}$ and $\mathbf{B}$ are bounded (as in our case where $\|\mathbf{B}\|=\|\mathbf{1}\|=1$ ).

The existence of traps in compartmental systems shows that robust mass balance does not exclude zero eigenvalues (2biA). As mentioned above, this excludes the possibility of ISS with respect to fixed points. However, in this work, we also consider ISS with respect to invariant sets. And while up to now those sets have always been regions of the phase space bounded in all directions, we can also look at the possibility of invariant sets as big as linear subspaces. Let us develop this idea starting from the following artificial example of a single pool without outflow:

$\dot{C}=0 C+I(t)$.

It is robustly mass balanced and, for $I=0$, stable in the sense of Lyapunov for all start values $C_{0}$; but of course unstable (in the sense of both Lyapunov and ISS) for all $I(t) \neq 0$ since it is a trap that never releases anything that went into it. Let us extend the system to a parallel two-pool model:

$$
\left(\begin{array}{l}
\dot{C}_{1} \\
\dot{C}_{2}
\end{array}\right)=\left(\begin{array}{ll}
0 & 0 \\
0 & -\lambda
\end{array}\right)\left(\begin{array}{l}
C_{1} \\
C_{2}
\end{array}\right)+\left(\begin{array}{l}
I_{1}(t) \\
I_{2}(t)
\end{array}\right) .
$$


Pool 1 is still a trap. Every point on the $C_{1}$ axis is a fixed point for the input-free system, but none of them is stable with respect to inputs with $I_{1}(t) \neq 0$. So we do not have any control about the $C_{1}$ values, but we do know that $C_{2} \rightarrow 0$ as $t \rightarrow \infty$. The $C_{1}$ axis is clearly an invariant set for $I_{2}(t)=0$ and it would be possible to show ISS with respect to this set. To further generalize and connect to the eigenvector properties, now consider this system to be the Jordan normal form of an originally given reservoir system like

$\left(\begin{array}{ll}-\frac{\lambda_{1}}{2} & \frac{\lambda_{2}}{2} \\ \frac{\lambda_{1}}{2} & -\frac{\lambda_{2}}{2}\end{array}\right)$,

with eigenvalues $\left\{0,-\lambda=-\frac{1}{2}\left(\lambda_{1}+\lambda_{2}\right)\right\}$. The original system will have an ISS invariant set given by the subspace spanned by the eigenvectors belonging to 0 (in the example $\left.\left(\frac{\lambda_{2}}{\lambda_{1}}, 1\right)^{T}\right)$. In case of more than one zero eigenvalue, an invariant set can be chosen as the span of all the eigenvectors belonging to a zero real part eigenvalue. As mentioned above (Jacquez and Simon 1993), every zero eigenvector corresponds to a simple trap. An example of a compartment model with a trap is given by the RothC model (Coleman and Jenkinson 1999). However, the trap in this case turns out to be completely disconnected from the rest of the system, receiving inputs neither from the outside nor from any other pool.

\section{General remarks on non-linear influx controlled systems}

We have already seen one example of a compartmental model that is not ISS. This is true for many compartmental systems. Even the subclass of systems governed by mass action kinetics provides a lot of unbounded examples (Angeli 2011). Actually, conservation of mass is not such a severe constraint on its own with respect to stability. Under the heading of "Anything can happen" (Theorem 4 Jacquez and Simon (1993)) show that every bounded autonomous $n$ dimensional system can be mapped to a $(n+1)$-dimensional compartmental system, which means that anything (ranging from GAS, over several equilibria, limit cycles to divergence and chaos) that can happen in a bounded autonomous system can also happen in compartmental systems. On the other hand, our closer look at (uniformly asymptotically stable) linear systems has revealed their remarkable property of a linear negative control feedback that stabilizes the equilibrium. It is easy to imagine non-linear systems that exhibit a similar or even stronger negative feedback. One can then conjecture that these systems are good candidates for ISS. It is usually much harder to prove this though.

We do not know of any general result guaranteeing ISS for influx controlled non-linear compartmental systems, not even for a subclass.
The question arises if there is some assistance in establishing the desired ISS result for a given system. To find an ISS Lyapunov function for our non-linear example (Eq. 10 et seq.), we conjectured that if we find a Lyapunov function for a linear system that exhibits a weaker feedback towards its invariant set than our non-linear system, then this Lyapunov function might still work for the non-linear case.

Accordingly, we derived the Lyapunov function for the linear system by the textbook procedure described below and then checked that the resulting Lyapunov candidate still worked for our non-linear, non-autonomous system.

To understand the approach for autonomous linear systems, let us first assume that the Lyapunov function can be expressed by a (yet unknown) quadratic form: $V(\mathbf{x})=$ $\mathbf{x}^{T} \mathbf{P x}$, where $\mathbf{x}$ is the deviation from the equilibrium. The derivative of $V$ along solutions is then given by $\dot{V}(\mathbf{x})=$ $\dot{\mathbf{x}}^{T} \mathbf{P x}+\mathbf{x}^{T} \mathbf{P} \dot{\mathbf{x}}=\mathbf{x}^{T} \mathbf{A}^{T} \mathbf{P x}+\mathbf{x}^{T} \mathbf{P A x}$. Next, we observe that a positive definite quadratic form $\mathbf{Q}$ fulfills $\mathbf{x}^{T} \mathbf{Q x} \leq \alpha|\mathbf{x}|^{2}$ for some $\alpha>0$. So if $\mathbf{P}$ solves the Lyapunov equation $\mathbf{A}^{T} \mathbf{P}+\mathbf{P A}-\mathbf{Q}=0$, this implies $\dot{V}=\mathbf{x}^{T} \mathbf{A}^{T} \mathbf{P x}+\mathbf{x}^{T} \mathbf{P A x}=$ $-\mathbf{x}^{T} \mathbf{Q x} \leq-\alpha|\mathbf{x}|^{2}$ for all $\mathbf{x}$. It is well known that such a $\mathbf{P}$ can be found for any positive definite $\mathbf{Q}$ if $\mathbf{A}$ is a Hurwitz matrix. It is also clear that any arbitrarily chosen positive definite $\mathbf{P}$ implies a positive definite $\mathbf{Q}$ with its largest eigenvalue $\alpha>0$. So we can choose $\mathbf{P}=\mathbf{1}$ implying $V=\mathbf{x}^{2}$. This most simple Lyapunov function will work for every stable LTI system. For non-linear systems, this is not certain, but still possible as proved for our first example. Note that $V=\mathbf{x}^{2}$, or more generally $V=\mathbf{x}^{T} \mathbf{P x}$, is also smooth, which is essential to prove ISS in contrast to GAS where we do not need this property.

Our example can also be seen as an instance of a more general heuristic approach towards a proof of ISS: One can first attempt to prove 0-GAS by means of a smooth Lyapunov function and and then try to reuse it for ISS.

We are therefore particularly interested in systems for which the construction of Lyapunov functions can be alleviated. An example is given by "port-controlled Hamiltonian systems." Due to their inherent mass balance, compartmental systems can always be written in this form with the total mass replacing the energy as the storage function. The exact translation can be found in Bastin (1999). The procedure to obtain a Lyapunov function described in Maschke et al. (2000) does, however, not succeed automatically. It fails for our example.

There are several more cases of reservoir systems for which 0-GAS can be proved by exploiting more special properties of the systems. For an overview, we point in particular to Jacquez and Simon (1993) and Bastin (1999) particularly the appendix of the later and the references therein. Unfortunately, the Lyapunov candidates are not always suitable for ISS since they are in many cases not smooth. If they are, one can use them as a starting point 
and check if they fulfill the additional requirements for an ISS Lyapunov function. Concluding, one can say that proving ISS for non-linear systems is difficult to achieve and will require knowledge of special properties that can be exploited. It would be somewhat misleading if we only presented examples where we know what to do.

However, the difficulty of establishing ISS for bigger subclasses of compartmental models with influxes is also revealing: Although the cumulative mass of a compartmental system is a Lyapunov function for the closed system (without influx), it does not remain one for a system with influx since there are clearly trajectories that at least temporarily increase the overall mass. This underlines that ISS is a different property than mass conservation. The input signal in influx controlled systems is of a different dimension (mass per time) than the conserved property. There is actually the related but fundamentally different concept of integrated input to state stability, iISS (Sontag 2008; 1998a), that is natural for the integrated influx. Implicitly, we already drew one conclusion from the iISS property: the conservation of mass we assumed from the start. Influx controlled systems that are ISS have to do more: instead of maintaining a bounded state for a bounded integrated influx (mass), they must be able to cope with just a bounded flux (mass per time). Clearly, not all compartmental systems can do this; some fill up.

The general idea behind the construction of Lyapunov functions is to exploit any conserved property (originally energy). In search of an ISS Lyapunov function, one should therefore also look beyond mass. In modeling and controlling continuous stirred tank reactors, entropy or other thermodynamic potentials are used (see for instance Hoang et al. (2011)). To mention an ecological example, we point to Appendix A4 of Bastin (1999), who studies a special conservative Lotka-Volterra system that is also compartmental.

\section{Zero deficiency networks driven by changes in reaction rates}

Up to now, the driving signal has always been the timedependent change in the influx of mass. We now present an example where the ISS framework can be used to study the effect of time-dependent temperature changes on flux rates. Instead of constructing an example from scratch, we use the opportunity to introduce a more general result (Chaves 2005), which is based on the theory of zero deficiency networks of chemical reactions (Horn and Jackson 1972; Feinberg 1977; 1987). A detailed explanation for the terms deficiency or zero deficiency can be found in Feinberg (1987). To show that it is a property easily checked and interpreted from an application-centered viewpoint (in this case chemical), we give an abbreviated definition.
A reaction network consists of complexes and reaction arrows between them. Not all complexes need to be connected by reaction arrows. A group of connected complexes is called a linkage class. The trajectories describing the evolution of concentrations of the species in the reactions are geometrically confined by stoichiometry. They can only evolve on the stoichiometric manifold. The dimension of this manifold is given by the number of the linear independent stoichiometric reaction vectors. It can be checked immediately by linear algebra after writing down the composition of the complexes with respect to the species in a matrix. Let $n$ denote the number of complexes, $l$ the number of linkage classes, and $s$ the dimension of the stoichiometric manifold. Then the deficiency $d$ of the network is given by $d=n-s-l$.

The stability of the system (in this case ISS) can be predicted by checking this essential chemical criterion. It would be extremely interesting to have such criteria based on physical or in our case even ecological properties of systems. When we started to compare compartmental systems with respect to their stability properties, we hoped that the conservation of mass might be such a property. We have already seen that this is not enough, and proving ISS turns out to be much more difficult. Actually, one needs all the help one can get. The result of Chaves (2005) provides such valuable assistance for a special kind of non-linear nonautonomous compartmental systems based on stoichiometry and therefore has potential for ecological applications. It incorporates mass action and Michaelis-Menten kinetics, which occur in ecological soil models (e.g., Wang et al. (2012) and Manzoni and Porporato (2007)). Its main limitation for reservoir models consists in its dependency on the zero deficiency property, which guarantees the existence of a unique non-boundary equilibrium, but is usually lost for reaction networks with mass influx from outside. While this fact renders a lot of applications more difficult, the result is still very general and potentially helpful as a starting point. All the underlying mathematical results, including the appropriately refined definition of ISS for this application and its proof, are developed in Chaves (2005) and are beyond the scope of this paper. However, the basic introduction we have given so far should make the original paper accessible.

While Chaves (2005) focuses on the biochemical example of receptor ligand complex formation, we give a minimal application that could represent processes taking place in the eutrophication of a lake. Consider the following chemical reactions:

$$
\begin{aligned}
\mathrm{C}_{6} \mathrm{H}_{12} \mathrm{O}_{6}+6 \mathrm{O}_{2} & \rightleftharpoons 6 \mathrm{CO}_{2}+6 \mathrm{H}_{2} \mathrm{O}, \\
\mathrm{CH}_{4}+12 \mathrm{O}_{2} & \rightleftharpoons 6 \mathrm{CO}_{2}+12 \mathrm{H}_{2} \mathrm{O} .
\end{aligned}
$$

By adding $6 \mathrm{H}_{2} \mathrm{O}$ to both sides of the first equation (a dilution), we can combine the two equations. The five 
species $\mathrm{C}_{6} \mathrm{H}_{12} \mathrm{O}_{6}, \mathrm{O}_{2}, \mathrm{CO}_{2}, \mathrm{H}_{2} \mathrm{O}$, and $\mathrm{CH}_{4}$ form the three complexes

$$
\begin{aligned}
\mathrm{I} & =\mathrm{C}_{6} \mathrm{H}_{12} \mathrm{O}_{6}+6 \mathrm{O}_{2}+6 \mathrm{H}_{2} \mathrm{O}, \\
\mathrm{II} & =6 \mathrm{CO}_{2}+12 \mathrm{H}_{2} \mathrm{O}, \\
\mathrm{III} & =\mathrm{CH}_{4}+12 \mathrm{O}_{2},
\end{aligned}
$$

and can be written in one equation:

$$
\mathrm{C}_{6} \mathrm{H}_{12} \mathrm{O}_{6}+6 \mathrm{O}_{2}+6 \mathrm{H}_{2} \mathrm{O} \underset{a_{2,1}}{\stackrel{a_{1,2}}{\rightleftharpoons}} 6 \mathrm{CO}_{2}+12 \mathrm{H}_{2} \mathrm{O} \underset{a_{3,2}}{\stackrel{a_{2,3}}{\rightleftharpoons}} \mathrm{CH}_{4}+12 \mathrm{O}_{2} .
$$

Assuming mass action kinetics and using the abbreviation $\left(C_{1}, C_{2}, C_{3}, C_{4}, C_{5}\right)^{T}=\left(C_{C_{6} H_{12} O_{6}}, C_{O_{2}}, C_{C_{2}}, C_{H_{2} O}\right.$, $\left.\mathrm{C}_{\mathrm{CH}_{4}}\right)^{T}$ for the concentrations, this leads to the following highly non-linear system:

$$
\left(\begin{array}{c}
\dot{C}_{1} \\
\dot{C}_{2} \\
\dot{C}_{3} \\
\dot{C}_{4} \\
\dot{C}_{5}
\end{array}\right)=\left(\begin{array}{c}
-C_{1} C_{4}^{6} C_{2}^{6} a_{2,1}(T)+C_{3}^{6} C_{4}^{12} a_{1,2}(T) \\
-6 C_{1} C_{4}^{6} C_{2}^{6} a_{2,1}(T)-12 C_{5}^{6} C_{2}^{12} a_{2,3}(T)+6 C_{3}^{6} C_{4}^{12} a_{1,2}(T)+12 C_{3}^{6} C_{4}^{12} a_{3,2}(T) \\
6 C_{1} C_{4}^{6} C_{2}^{6} a_{2,1}(T)+6 C_{5}^{6} C_{2}^{12} a_{2,3}(T)-6 C_{3}^{6} C_{4}^{12} a_{1,2}(T)-6 C_{3}^{6} C_{4}^{12} a_{3,2}(T) \\
6 C_{1} C_{4}^{6} C_{2}^{6} a_{2,1}(T)+12 C_{5}^{6} C_{2}^{12} a_{2,3}(T)-6 C_{3}^{6} C_{4}^{12} a_{1,2}(T)-12 C_{3}^{6} C_{4}^{12} a_{3,2}(T) \\
-6 C_{5}^{6} C_{2}^{12} a_{2,3}(T)+6 C_{3}^{6} C_{4}^{12} a_{3,2}(T)
\end{array}\right),
$$

where $T$ is temperature and $a_{i, j}(T)$ a temperaturedependent rate. If we assume that $T$ is time dependent, the system becomes non-autonomous. Just looking at the definitions in Feinberg (1987), one finds that there are three complexes. The stoichiometric space of this example is of dimension two, the number of linkage classes is one, and the deficiency is equal to zero. Therefore, Feinberg (1987) ensures that a non-boundary equilibrium exists, while Chaves (2005) proves that it is ISS.

To demonstrate the signature behavior of an ISS system by numerical experiments (Fig. 3), we choose the following

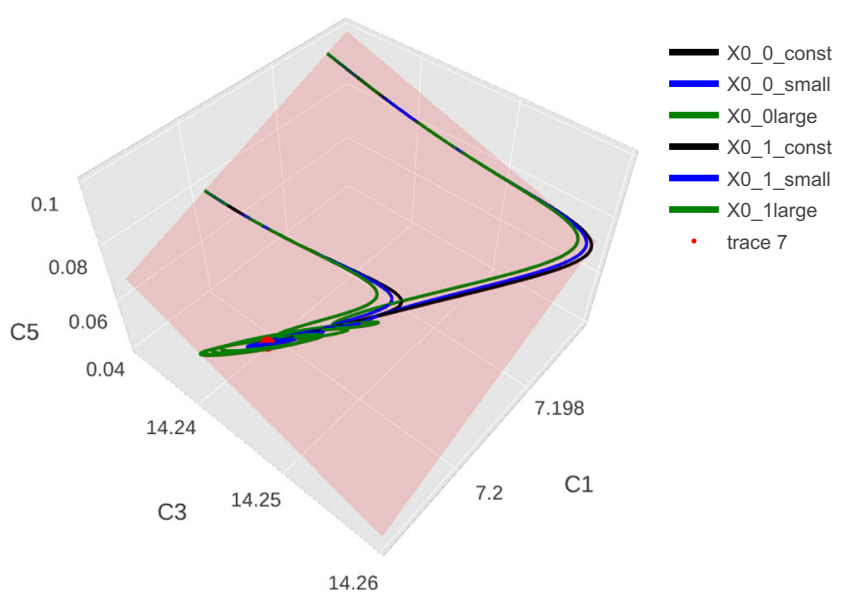

Fig. 3 Three-dimensional projection of the five-dimensional phase portrait. For each of the two start vectors, we show three trajectories: black undisturbed, blue with small temperature variation, green with large temperature variation. The fixed point of the undisturbed system is visible as red dot. The two disturbed solutions can be seen to stay in neighborhoods depending on the size of the disturbance. Due to the stoichiometric constraints, all trajectories of the example system are confined to a two-dimensional stoichiometric manifold in $\mathbb{R}^{+^{5}}$. The projection to the three plotted dimensions $C_{1}, C_{3}$, and $C_{5}$ is made visible as transparent red plane
Arrhenius temperature dependence for the rates: $a_{i, j}=$ $F_{i, j} e^{-\frac{E_{i, j}}{R T}}$, with $\left\{T: \frac{\Delta_{T}}{2} \sin (\omega t)+T_{0}\right\}$.

Remarks:

It is worth mentioning that the Lyapunov function used by Chaves (2005) and motivated by Sontag (2001) is related to entropy considerations and very different from the examples encountered so far.

\section{A non-compartmental multi-stable example}

In the introduction, we referred to critical regime changes and showed by a simple example that classical stability analysis (for autonomous systems) cannot guarantee stability with respect to time-dependent perturbations at all. If such a result is required, one has to turn to non-autonomous concepts such as ISS.

Since our examples only had one invariant set, the discussion of regime changes could have used ISS only to exclude the possibility of such critical transitions. In contrast to classical stability, which provides a safeguard against perturbations of the state or (by bifurcation analysis) uncertainty with respect to parameters, ISS also excludes regime changes caused by a bounded external forcing.

We also mentioned that the ISS framework can be used to study systems that are known to exhibit critical transitions and pointed to the work of Chaves et al. (2008), where a local version of ISS is developed and used.

We cited Angeli and Efimov (2015) for an even more general application of ISS to multi-stable systems. It would be nice to have a meaningful multi-stable ecological reservoir example for which we could prove ISS by a very elegant argument, short enough for this paper. While this is unfortunately not the case, we did find the following conceptual example in Angeli and Efimov (2015), which may 
be very relevant from an ecological viewpoint. Consider the one-dimensional system:

$\dot{x}=-x^{5}+x^{3}+x u$.

The unperturbed system exhibits a set of three equilibria $\mathcal{S}=\{-1,0,-1\}$ of which $\{-1,1\}$ are asymptotically stable and 0 is unstable. The standard ISS framework that we used so far can provide ISS for the (compact) interval $\mathcal{A}=[-1,1]$ by the Lyapunov function $V=|x|_{\mathcal{A}}^{2}$. Instead, Angeli and Efimov (2015) show a generalized version of the AG property using the Lyapunov function $V(x)=(x-1)^{2}(x+1)^{2}$ for the non-compact set $\mathcal{S}$. The three-element set $\mathcal{S}$ is much more interesting because the three distinct equilibria can now be resolved. Furthermore, the numerical experiment (Fig. 4) suggests that the inner equilibrium 0 which was repelling for small perturbations becomes attractive when the size of the perturbation increases.

This example could be prototypical for an ecosystem that can switch into a high perturbation compensation mode that is not visible as long as the perturbations are small.

\section{Remark:}

We do not explain this behavior, but only show that it is possible for an ISS system. This is not only a different kind of stability, but also a different kind of regime change not predictable by classical stability analysis. It underlines that non-autonomous systems have to be discussed differently.

\section{Cascades of ISS systems}

A feature of ISS systems very much valued in control theory is the fact that cascades of ISS systems are themselves ISS (Sontag 1989). If this kind of decomposition is possible, proofs can be greatly simplified. For those compartmental models where energy or matter flows only in one direction between subsystems, this structure is implied automatically.

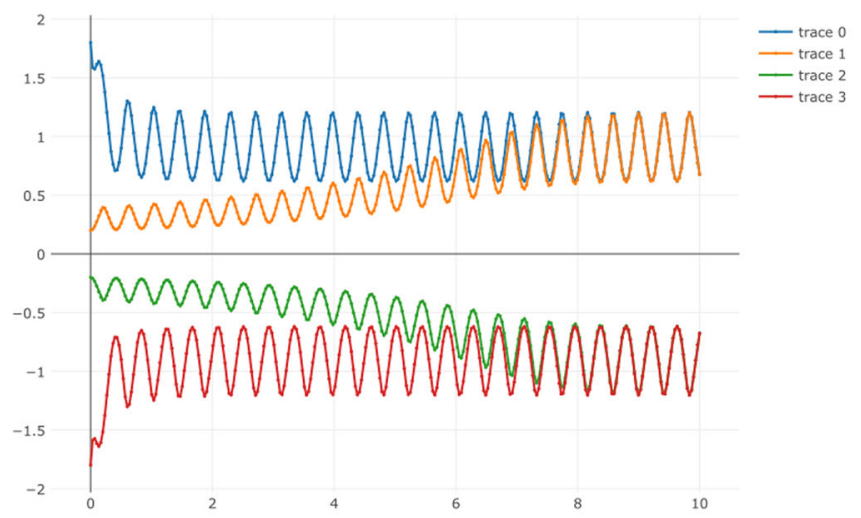

Fig. 4 Simulation results for Eq. 24, four different initial conditions, and two different perturbations: $u_{1}(t)=5 \sin (15 t)$ (left), $u_{2}(t)=35 \sin (15 t)$ (right). The bigger perturbation $u_{2}$ "stabilizes"
In such cases, it suffices to show ISS for the subsystems. Examples could be the RothC model (Coleman and Jenkinson 1999) (which can also be proved to be ISS using its linearity) and possible non-linear variants of it. Cascades are represented by systems like the following:

$$
\left(\begin{array}{l}
\dot{z}_{1} \\
\vdots \\
\dot{z}_{n} \\
\dot{x}_{1} \\
\vdots \\
\dot{x}_{m}
\end{array}\right)=\left(\begin{array}{l}
f_{1}\left(z_{1} \cdots z_{n}, x_{1}, \cdots x_{m}\right) \\
\vdots \\
f_{n}\left(z_{1} \cdots z_{n}, x_{1}, \cdots x_{m}\right) \\
g_{1}\left(x_{1} \cdots x_{m}, u_{1}, \cdots u_{l}\right) \\
\vdots \\
g_{m}\left(x_{1} \cdots x_{m}, u_{1}, \cdots u_{l}\right)
\end{array}\right) .
$$

An interesting corollary (Chapter 4 Sontag (2008)) is that the cascade of a GAS $\mathbf{x}$ system with an ISS $\mathbf{z}$ system is GAS. This recommends ISS as a tool to prove GAS for composite ecosystem models.

\section{Conclusions and outlook}

\section{What ISS offers}

We have seen that the ISS concept provides a notion of stability applicable to the situation we naturally find ourselves in when dealing with ecological dynamical systems: driving variables that change with time and a very limited hope to find the system in equilibrium. While the former requirement can be met by the extensions of concepts like stable fixed points or invariant sets to the realm of nonautonomous systems, the latter ability to describe "stability" for non-equilibrium situations sets it apart from the standard concepts. It makes ISS a flexible enough stability concept to describe the ability of the system to return to a normal mode of operation, a property related to the ecological concept of "resilience," originally introduced as alternative to stability (Holling 1973).

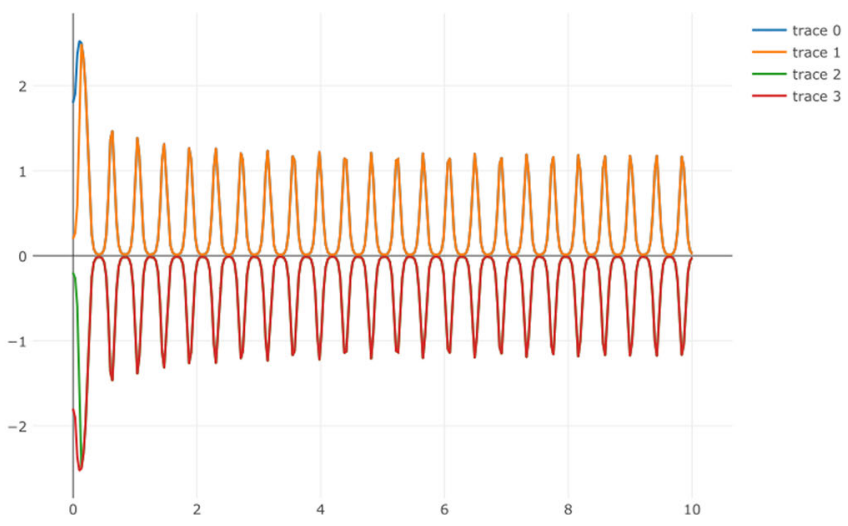

the solution around the unstable fixed point at $x=0$. The solutions that were previously attracted to either -1 or 1 are now attracted to 0 
From a conceptual viewpoint, ISS ensures that a system recovers eventually. However, the speed of this recovery will also frequently be interesting for the practical analysis of an ecosystem. Although at a first glance ISS as an asymptotic concept seems to ignore recovery speeds, this interesting information is contained in the specific comparison functions $\beta$ and $\gamma$ necessary for the proof. These functions also provide upper boundaries for possible states of the system. These estimates can be sharpened by a careful construction of minimal $\beta$ and $\gamma$.

\section{Relation to Sobolev spaces and stochastic approaches}

The fact that the ISS definition does not put limits on how fast the input changes connects it to two other approaches. First, it is clear that there is room for other-in terms of their requirements-weaker concepts that guarantee stability only under more specific conditions about the smoothness of the input. It is not difficult to imagine ecological systems that can handle a certain speed of change, but break if the changes occur too rapidly. The related concept of $D^{k} I S S$, an extension with respect to Sobolev spaces, has not been discussed here, but can be found, (e.g., in Sontag 2008, section 6) or Angeli et al. (2003).

Second, since ISS does not require that $\mathbf{u}$ is smooth, it can incorporate signals such as Brownian motion. ISS estimates still hold in the case where $\mathbf{u}$ represents an uncertainty. In this respect, ISS provides deterministic limits of the results of uncertain stochastic inputs.

In another respect, ISS can be extended to stochastic differential equations (SDEs) in particular to the notion of SISS (Liu et al. 2008) where it generalizes the concept of a solution process bounded in probability in the same way the analytic ISS generalizes GAS. Since the discussion of SDEs and their relations to ISS is technically far beyond the scope of this work, we point to Tsinias (1998) and Liu et al. (2008) for an introduction and many further references.

\section{ISS for compartmental systems}

By supplying a counter example (16), we illustrated that ISS is not an automatic result of natural properties of reservoir systems like mass balance, but rather a quite distinguishing feature of systems robustly stabilized by some additional regulating process or principal. Proving ISS for a given arbitrary compartmental system turned out to be very difficult. Our only successful applications to nontrivial examples are either linear or based on zero deficiency. While the linear models could have been discussed completely without ISS from an operator-centered perspective, ISS provides a mapping of these results into the domain of state space techniques dominating the analysis of non-linear systems. We would not have been able to grasp the concept of "region of stability" in any precise way even for linear systems without ISS. We also showed that ISS cascades can be built from ISS subsystems easily for unidirectional fluxes between them. The chances to prove ISS also increase if one chooses an appropriately (large) invariant set, including cases in which ISS might be shown on a quotient space or for a subset of the model equations.

\section{Outlook}

In the future, we would like to see ISS applied to modern non-linear ecological models. However, even GAS can be difficult to prove (or disprove) as the following tiny example

$$
\left(\begin{array}{l}
\dot{C}_{1} \\
\dot{C}_{2}
\end{array}\right)=\left(\begin{array}{l}
I+k_{2} C_{2}-k_{1} \frac{C_{1}}{K_{m}+C_{1}} C_{2} \\
-k_{2} C_{2}+r k_{1} \frac{C_{1}}{K_{m}+C_{1}} C_{2}
\end{array}\right),
$$

with constants $I, k_{1}, k_{2}, K_{M}>0$ and $0<r<1$ shows (Wang et al. 2013). It is not covered by any of the stability criteria in Jacquez and Simon (1993) or Bastin (1999) for compartmental systems.

Acknowledgments Open access funding provided by Max Planck Society. We would like to thank H. Metzler very much for repeated conscientious proofreadings and many helpful comments, regarding the language as well as the mathematical content.

\section{Compliance with Ethical Standards}

Funding Funding was provided by the Max Planck Society and the German Research Foundation through its Emmy Noether Program (SI 1953/2-1).

Open Access This article is distributed under the terms of the Creative Commons Attribution 4.0 International License (http:// creativecommons.org/licenses/by/4.0/), which permits unrestricted use, distribution, and reproduction in any medium, provided you give appropriate credit to the original author(s) and the source, provide a link to the Creative Commons license, and indicate if changes were made.

\section{Appendix 1. Stability concepts}

\subsection{Stability notions for autonomous systems}

In Eq. 2, there is no explicit time dependency, so we are in the realm of classical autonomous dynamical systems. The search for invariants naturally leads to the notion of invariant sets (Definition 2). One can now look for minimal invariant sets. This leads to the special case of an invariant set with only one element, better known as a fixed point (FP) and often used interchangeably with the terms equilibrium or steady state

$\mathcal{A}=\left\{\overline{\mathbf{x}}_{\mathbf{p}}\right\}$ with $\mathbf{f}\left(\overline{\mathbf{x}}_{\mathbf{p}}, \mathbf{p}\right)=0$.

Examples of larger minimal invariant sets are limit cycles (two-dimensional), limit tori (three-dimensional), or even strange attractors. 
In the presence of invariant sets, we can ask if they are stable with respect to perturbations $\Delta \mathbf{x}$. Stability can be defined precisely by the combination of Lyapunov stability and attractiveness, which are usually stated for FPs, but here immediately generalized to invariant sets.

Definition 13 (Local Lyapunov stability of an invariant set) Consider system (2). Suppose $\mathcal{A}$ is an invariant set of the system. Then $\mathcal{A}$ is said to be locally Lyapunov stable if

for all $\varepsilon>0 \exists \delta:\left|\mathbf{x}_{0}\right|_{\mathcal{A}}<\delta \Longrightarrow|\mathbf{x}(t)|_{\mathcal{A}}<\varepsilon$, for all $t>0$,

with the set distance defined as

$|\mathbf{x}|_{\mathcal{A}}=\inf _{y \in \mathcal{A}}|\mathbf{x}-\mathbf{y}|$.

Definition 14 (Asymptotic stability and basin of attraction) Let $\mathcal{A}$ be a compact invariant set. If $\mathcal{A}$ is Lyapunov stable, then the set

$\mathcal{B}=\left\{\mathbf{x}_{0}: \lim _{t \rightarrow+\infty}\left|\mathbf{x}\left(t, \mathbf{x}_{0}\right)\right|_{\mathcal{A}}=0\right\}$

is called the basin of attraction.

If $\mathcal{B}=\mathbb{R}^{n}$, we call $\mathcal{A}$ globally attractive and the combination of Lyapunov stability and global attractiveness, global asymptotic stability (GAS). In Euclidean space, the only minimal compact invariant sets (attractors) with GAS are equilibria (Angeli 2004). Interestingly, the basin of attraction of an invariant set is itself a (maximal) invariant set.

We can formulate GAS also using comparison functions which will prove useful later:

$\exists \beta \in \mathcal{K} \mathcal{L}:\left|\mathbf{x}\left(t, \mathbf{x}_{0}\right)\right|_{\mathcal{A}} \leq \beta\left(\left|\mathbf{x}_{0}\right|_{\mathcal{A}}, t\right)$, for all $\mathbf{x}_{0}$.

The notion of global attractiveness is natural for some systems (for instance linear ODEs), but in general, a rather strong requirement, in particular for multi-stable systems (with more than one stable equilibrium). The only invariant set with global asymptotic stability has to include those equilibria and thus may become rather too large to answer questions regarding the long-term behavior of the system in sufficient detail. See Angeli and Efimov (2015) for recent results allowing a more detailed analysis, which exceeds the formal scope of this article.

With the definitions introduced so far, we are well equipped to study invariant sets of Eq. 2 and their stability for any fixed set of parameters $\mathbf{p}=\left(p_{1}, p_{2}, \cdots, p_{n}\right)^{T}$. We can also ask how existence and stability change with respect to those parameters. For example, does the number of invariant sets change (bifurcations), do stable ones become unstable or vice versa? How do the basins of attraction change? See for instance Manzoni and Porporato (2007) as a recent example addressing all those questions.
Now, let us consider the parameters to be functions of time $p_{1}(t), p_{2}(t), \cdots, p_{n}(t)$. This adds a whole new level of complexity. While Eq. 2 is called dynamical with respect to $\mathbf{x}$, it is static with respect to $p_{1}, p_{2}, \cdots, p_{n}$. While faithfully representing the rate of change of $\mathbf{x}$, it completely misses the influence the rate of change of $p_{1}, p_{2}, \cdots, p_{n}$ might have. Therefore, answers to questions we asked so far will only be useful if the quasi-static approximation

$|\dot{\mathbf{p}}| \ll|\dot{\mathbf{x}}|$

is justified. Otherwise, they can be extremely misleading, as example (3) in the introduction showed.

\subsection{Generalizations to non-autonomous systems}

Looking at Eq. 6, we can formulate two aspects of stability for time-varying systems. In this paragraph, we still consider invariant sets maintained for all times (despite the driving forces represented by the time-varying terms) so that, although the system is driven, it is not driven away from the fixed point or invariant set. In this scenario, one possibility is to just include time in the above definitions. Stability becomes dependent on the start time $t_{0}$, since it may vanish or appear at certain points in the history of the system.

Definition 15 (Lyapunov stability and uniform Lyapunov stability for non-autonomous systems) Consider a nonautonomous non-linear dynamical system

$\dot{\mathbf{x}}=\mathbf{f}(\mathbf{x}, t), \quad \mathbf{x}\left(t_{0}\right)=\mathbf{x}_{0}$.

Suppose $\mathbf{f}$ has an invariant set $\mathcal{A}$, then this set is said to be Lyapunov stable at time $t_{0}$ if for all $\varepsilon>0$ exists a $\delta$ such that:

$\left|\vec{x}\left(t_{0}\right)\right|_{\mathcal{A}}<\delta \Longrightarrow\left|\vec{x}\left(t, \vec{x}_{0}, t_{0}\right)\right|_{\mathcal{A}}<\varepsilon$, for all $t>t_{0}$.

$\mathcal{A}$ is called uniformly stable if $\delta$ is not a function of $t_{0}$ so that Eq. 29 holds for all $t_{0}$.

A similar alteration of Eq. 26 leads to the notion of uniform local attractiveness. The combination of uniform Lyapunov stability and local uniform attractiveness leads to the important notion of uniform asymptotic stability (UAS). As before, there is a global version that admits a comparison function formulation.

Definition 16 (Global uniform asymptotic stability (GUAS)) Consider system (28). The invariant set $\mathcal{A}$ is uniformly globally asymptotically stable if for all trajectories $\mathbf{x}\left(t, \mathbf{x}_{0}, t_{0}\right)$ there exists a $\beta \in \mathcal{K} \mathcal{L}$ such that

$\left|\mathbf{x}\left(t, \mathbf{x}_{0}, t_{0}\right)\right|_{\mathcal{A}} \leq \beta\left(\left|\mathbf{x}_{0}\right|_{\mathcal{A}}, t-t_{0}\right)$, for all $t_{0}$. 


\section{Appendix 2. Reservoir models}

We treat here reservoir, pool, and compartmental systems as synonymous and use relevant definitions from Jacquez and Simon (1993) and references therein.

\subsection{Properties and definition}

Definition 17 (Compartmental system) Let $F_{i, j}$ be the flux from pool $j$ to pool $i$, for all $i, j \neq i \in\{1 \ldots n\}, F_{i, 0}=$ $I_{i}$ define the external influx to pool $i$ and $F_{0, i}=O_{i}$ the external outflux from pool $i$ (Fig. 5).

If $F_{i, j}(\mathbf{C}, t) \geq 0$ for all $i, j \in\{0 \ldots n\}$ and

$C_{j}=0 \Longrightarrow F_{i, j}(\mathbf{C}, t)=0$

we call the ODE system

$\dot{C}_{i}=\sum_{j=0 . i \neq j}\left(-F_{j, i}(\mathbf{C}, t)+F_{i, j}(\mathbf{C}, t)\right) \quad$ for all $i$

compartmental.

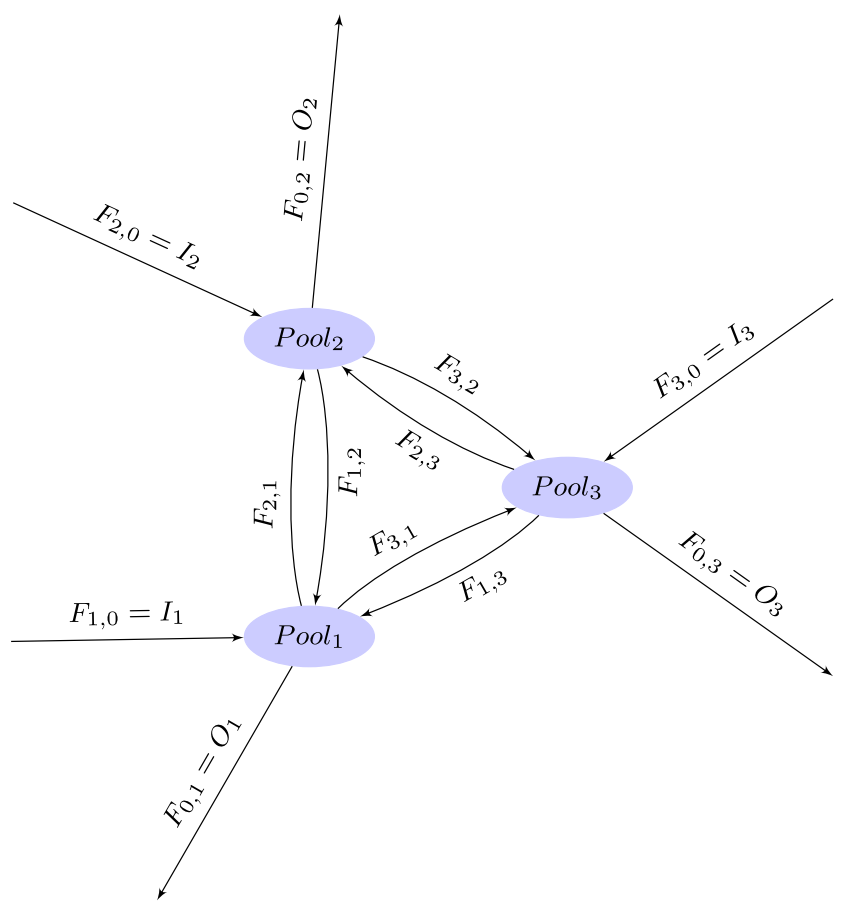

Fig. 5 A compartmental model with three pools and all possible connections between the pools and the environment. Note that the flux between pool $_{k}$ and pool $_{l}$ is considered a property of the pipeline, meaning the flux from pool $_{k}$ into the pipeline is the same as the flux out of the pipeline into pool $_{k}$. This ensures mass balance for all fluxes and pools and also for the model as a whole, and makes this the prototype or normal form of mass-balanced models with internal connections. Every model that can be drawn in this form is mass balanced. Examples are not limited to networks of pipelines and reservoirs of fluids. Instead of a fluid, the total amount of a chemical element in different chemical substances (represented by the pools) can be described by it
Remarks:

1. The conditions guarantee mass balance, non-negative fluxes, and ensure that there cannot be any flux out of an empty pool. For non-negative initial values $\mathbf{C}_{0} \in \mathbb{R}^{+^{n}}$, the pool contents stay non-negative for $t \geq t_{0}$.

2. This is the standard definition of a compartmental system as in Jacquez and Simon (1993) and sufficient for all the examples in this paper. However, it is easy to imagine generalizations still treatable by the ISS framework. The compartment contents $\mathbf{C}$ could for instance depend on additional state variables: let $\mathbf{Y}$ be a vector of variables not describing contents of pools and consider the following system.

$\dot{C}_{i}=\sum_{j=0 . i \neq j}\left(-F_{j, i}(\mathbf{C}, \mathbf{Y}, t)+F_{i, j}(\mathbf{C}, \mathbf{Y}, t)\right)$ for all $i$ $\dot{\mathbf{Y}}=\mathbf{G}(\mathbf{C}, \mathbf{Y}, t)$

This includes (by transformation) higher order ODEs for $\mathbf{Y}$ as shown in Appendix 2.3, Eq. 47. Although the present work is only concerned with ISS, and therefore based on ODEs, it should be clear that even simple natural models of fluxes between reservoirs can be more complex than a formulation as a system of differential equations permits. Fluxes could for instance depend on the history of the system. Pool contents do not have to be continuous functions of time, let alone differentiable. Concluding, Definition 17 is widely applicable but not exhaustive.

3. Note that the form of Definition 17 does not have to be explicitly used in a model to ensure mass balance, but it must be possible to reformulate the model in this way. For example, in the general soil model of Sierra and Müller (2015), we can derive the fluxes through the metaphorical pipelines from the formulation shown in Appendix 2.2. Further examples fitting the robust mass balance definition are those describing the cycling of a chemical element, for instance carbon balance in plants or ecosystems (see, e.g., Luo and Weng (2011), Sierra and Müller (2015), Rasmussen et al. (2016)).

4. Property (31) in Definition 17 encourages to introduce $f_{i, j}$ defined by $F_{i, j}(\mathbf{C}, t)=f_{i, j}(\mathbf{C}, t) C_{i}$ and $f_{i, i}=-\left(f_{0, i}+\sum_{j \neq i} f_{j, i}\right)$ and write (32) in matrix form.

$\dot{\mathbf{C}}=\mathbf{f}(\mathbf{C}, t) \mathbf{C}+\mathbf{I}(t)$

A proof that $F_{i, j} \in C^{k} \Longrightarrow f_{j, i} \in C^{k-1}$ can be found in Appendix 1 of Jacquez and Simon (1993). This leads to the following definition. 
Definition 18 (Compartmental matrix) We call the matrix $\mathbf{f}$ in Eq. 33 compartmental if:

$$
\begin{aligned}
& f_{i, i} \leq 0 \text { for all } i, t \geq 0, \\
& f_{i, j} \geq 0 \text { for all } i \neq j, t \geq 0, \\
& \sum_{i=1}^{n} f_{i, j}=\sum_{i \neq j} f_{i, j}+f_{j, j}=-f_{0, j} \leq 0 \text { for all } j, t \geq 0
\end{aligned}
$$

Property (36) implies that $-f_{j, j} \geq \sum_{i \neq j} f_{i, j}$, for all $j$ for every compartment system. If we also know that $\mathbf{f}$ is nonsingular, it follows (equation (9) Jacquez and Simon (1993)) that $\mathbf{f}$ is diagonally dominant, i.e.,

$\exists d_{1}, \ldots, d_{n}>0$ such that $-d_{j} f_{j, j}>\sum_{i \neq j} d_{i} f_{i, j}$, for all $j$.

If we know (e.g., by physical, chemical, or biological arguments) that every compartment has an external outflow that never vanishes, Eq. 36 becomes a strict inequality, and the matrix $\mathbf{f}$ is strictly diagonally dominant. This is a very useful special case which can be directly exploited to prove ISS for linear time-varying systems. For the general SOM decomposition model in Appendix 2.2, strict diagonal dominance follows if $\mathbf{N}(\mathbf{C}, t) \mathbf{R}(\mathbf{C}, t)_{i, i}>0$, for all $i, t \geq 0$, which means that all pools respire at all times at least slightly.

Definition 19 (Strictly diagonally dominant compartmental matrix) We call a compartmental matrix $\mathbf{f}$ in Eq. 33 strictly diagonally dominant if

$\sum_{j=1}^{n} f_{i, j}=\sum_{j \neq j} f_{i, j}+f_{i, i}=-f_{0, i}<0$, for all $i, t \geq 0$

\subsection{General SOM decomposition model}

In Sierra and Müller (2015), we derived a formula generalizing soil organic matter decomposition models (e.g., Henin and Dupuis (1945), Andren and Kätterer (1997), Coleman and Jenkinson (1999), Parton et al. (1987), Fontaine and Barot (2005), Schimel and Weintraub (2003), Sinsabaugh and Follstad Shah (2012), Allison et al. (2010), Zelenev et al. (2000), Wang et al. (2013), and Manzoni and Porporato (2007)) using a system of the form

$$
\frac{d}{d t} \mathbf{C}=\mathbf{I}+\mathbf{T N} \mathbf{C} \text {. }
$$

In the derivation of Eq. 38, we did not refer to the pipeline metaphor explicitly. Therefore, we now express the fluxes $F_{j, i}$ in terms of the matrices $\mathbf{T}$ and $\mathbf{N}$, which shows that they have the desired properties. We will proceed as follows:

1. We divide the total inputs to pool $i$ between the external $I_{\text {ext }, i}$ coming from outside the system boundaries and internal input $I_{i n t, i}$ coming from other pools within the system. Similarly, we split the total output from this pool between the output $O_{\text {ext }, i}$ directly leaving the system (for instance by respiration) and $O_{i n t, i}$ the output of pool $i$ to be distributed to other pools in the system.

$\frac{d C_{i}}{d t}=I_{e x t, i}+I_{i n t, i}-O_{i n t, i}-O_{e x t, i}$, for all $i$

2. We express $I_{\text {ext }, i}, I_{i n t, i}, O_{\text {ext }, i}, O_{\text {int }, i}$ by $F_{i, j}$ and alternatively by the building blocks of $\mathbf{N}$ and $\mathbf{T}$.

$I_{\text {ext }, i}=F_{i, 0}$,

$I_{\text {int }, i}=\sum_{j=1, j \neq i}^{m} F_{i, j}$,

$O_{\text {ext }, i}=F_{0, i}$,

$O_{i n t, i}=\sum_{j=1, j \neq i}^{m} F_{j, i}$

Substituting in Eq. 40 yields

$\frac{d C_{i}}{d t}=F_{i, 0}+\sum_{j=1, j \neq i}^{m} F_{i, j}-\sum_{j=1, j \neq i}^{m} F_{j, i}-F_{0, i}$, for all $i$.

which is Eq. 32. We now show how the terms $I_{e x t, i}, I_{i n t, i}, O_{e x t, i}, O_{i n t, i}$ were constructed in Sierra and Müller (2015):

$\mathbf{I}_{i n t}=\mathbf{D O}_{i n t}$

where the distribution matrix $\mathbf{D}$ has a zero diagonal and also fulfills $\sum_{i} D_{i, j}=1$ expressing the fact that all internal outputs from source pools are distributed among the sink pools. Also note that inputs, and therefore the distribution coefficients, are non-negative $D_{i, j} \geq 0$ for all $i, j$.

This expresses the fluxes between the pools in terms of internal outputs. To describe the split of the total output into internal and external, we defined for every pool the proportion $r_{i}$ of output of pool $i$ released from the system. This permits fluxes out of the model as well as internal ones to be expressed in terms of total fluxes out of the pool

$F_{0, i}=r_{i} O_{i}$,

$F_{j, i}=D_{j, i}\left(1-r_{i}\right) O_{i}$.

Writing this in diagonal matrix form, we have $\mathbf{R}=\left(R_{i, i}\right)$ with $0 \leq R_{i, i}=r_{i} \leq 1$

$\mathbf{O}_{\text {ext }}=\mathbf{R O}$,

$\mathbf{O}_{i n t}=(\mathbf{1}-\mathbf{R}) \mathbf{O}$.

The internal outputs are expressed by means of the diagonal matrix $\mathbf{N}$, describing the per pool processing:

$\mathbf{O}=\mathbf{N C}$. 
Combining Eqs. 40, 41, 42, 43 and dropping the subscript ext from external inputs yields

$$
\begin{aligned}
\frac{d \mathbf{C}}{d t} & =\mathbf{I}+\mathbf{D} \mathbf{O}_{i n t}-\mathbf{O}_{i n t}-\mathbf{O}_{e x t} \\
& =\mathbf{I}+(\mathbf{D}-\mathbf{1}) \mathbf{O}_{i n t}-\mathbf{O}_{e x t} \\
& =\mathbf{I}+(\mathbf{D}-\mathbf{1})(\mathbf{1}-\mathbf{R}) \mathbf{O}-\mathbf{R O} \\
& =\mathbf{I}+((\mathbf{D}-\mathbf{1})(\mathbf{1}-\mathbf{R})-\mathbf{R}) \mathbf{O} \\
& =\mathbf{I}+((\mathbf{D}-\mathbf{1})(\mathbf{1}-\mathbf{R})-\mathbf{R}) \mathbf{N C} \\
& =\mathbf{I}+\mathbf{T N C} .
\end{aligned}
$$

Here $\mathbf{1}$ is the identity matrix, and $\mathbf{T}$ contains -1 in the main diagonal and transfer coefficients among pools in the offdiagonal entries. A very useful characteristic of $\mathbf{T}$ is the relationship of its columns to the release coefficients

$r_{j}=R_{j, j}=-\sum_{i} T_{i, j}$.

So it is possible to reconstruct the matrix $\mathbf{R}$ from $\mathbf{T}$ and thus also $\mathbf{D}$, so that we can express the fluxes through all the pipelines. We can connect this to Eq. 17 by choosing some positive $\mathbf{C}_{f}$ and using coordinate transformations, we set $\mathbf{x}(t)=\mathbf{C}(t)-\mathbf{C}_{f}, \mathbf{u}(t)=\mathbf{I}(t)-\mathbf{A}(t) \mathbf{C}_{f}, \mathbf{A}(t)=\mathbf{T}(t) \mathbf{N}(t)$, $\mathbf{B}(t)=\mathbf{1}$.

\subsection{Higher order systems encompassing reservoir models}

Take the extremely simplified model of an oscillation of the complete material between two pools with constant total mass.

$$
\begin{aligned}
\ddot{y} & =-y, \\
\dot{C}_{1} & =y, \\
\dot{C}_{2} & =-y .
\end{aligned}
$$

For sensible initial values (e.g., $t_{0}=0, y_{0}\left(t_{0}\right)=0, \dot{y}\left(t_{0}\right)=$ $\left.1, C_{1}\left(t_{0}\right)=0, C_{2}\left(t_{0}\right) \geq \int_{0}^{\pi \sin (\tau) d \tau}\right)$, the solutions $C_{1}(t)$ and $C_{2}(t)$ describe oscillating non-negative pool contents. It is also possible to enforce the compartmental character of the subsystem $\left(C_{1}, C_{2}\right)^{T}$ by writing

$$
\begin{aligned}
\ddot{y} & =-y, \\
\dot{C}_{1} & =F_{1,2}-F_{2,1}, \\
\dot{C}_{2} & =F_{2,1}-F_{1,2},
\end{aligned}
$$

with

$$
\begin{aligned}
& F_{1,2}(\mathbf{C}, y)= \begin{cases}y & \text { if } C_{2}>0, y>0, \\
0 & \text { otherwise },\end{cases} \\
& F_{2,1}(\mathbf{C}, y)= \begin{cases}y & \text { if } C_{1}>0, y>0, \\
0 & \text { otherwise }\end{cases}
\end{aligned}
$$

This example shows that even if an ODE system is not compartmental as a whole, it might well contain subsystems that are. The ISS concept is not limited to compartmental systems and could be applied here.

\section{Appendix 3. Coordinate transformations}

In the literature, ISS related definitions are often formulated by means of comparison functions, which are centered around an equilibrium value of zero. However, this is no restriction of generality since the following transformation is always possible.

\subsection{General non-linear systems}

Consider our general system:

$\dot{\mathbf{C}}=\mathbf{F}(t, \mathbf{C}, \mathbf{I}(t))$.

Suppose (47) has a fixed point $\mathbf{C}_{f}$. When we introduce a new variable $\mathbf{x}=\mathbf{h}(\mathbf{C})=\mathbf{C}-\mathbf{C}_{f}$, we arrive at a system

$\dot{\mathbf{x}}=\tilde{\mathbf{F}}(t, \mathbf{x}, \mathbf{I}(t))$,

with $\tilde{\mathbf{F}}(t, \mathbf{x}, \mathbf{I})=\mathbf{F}\left(t, \mathbf{x}+\mathbf{C}_{f}, \mathbf{I}(t)\right)$ and fixed point $\mathbf{x}_{f}=\mathbf{0}$. Furthermore, consider a similar coordinate translation for the input

$\mathbf{I}=\mathbf{I}_{0}(t)+\mathbf{u}(t)$.

We write

$\dot{\mathbf{x}}=\mathbf{f}(t, \mathbf{x}, \mathbf{u}(t))$,

with $\mathbf{f}(t, \mathbf{x}, \mathbf{u}(t))=\tilde{\mathbf{F}}\left(t, \mathbf{x}, \mathbf{u}(t)+\mathbf{I}_{0}(t)\right)$.

\section{References}

Allison SD, Wallenstein MD, Bradford MA (2010) Soil-carbon response to warming dependent on microbial physiology. Nat Geosci 3(5):336-340. 10.1038/ngeo846

Anderson DH (2013) Compartmental modeling and tracer kinetics, vol 50. Springer Science \& Business Media

Andren O, Kätterer T (1997) ICBM: the introductory carbon balance model for exploration of soil carbon balances. Ecol Appl 7(4):1226-1236

Angeli D (2004) An almost global notion of input-to-state stability. IEEE Trans Autom Control 49(6):866-874

Angeli D (2011) Boundedness analysis for open chemical reaction networks with mass-action kinetics. Nat Comput 10(2):751-774

Angeli D, Efimov D (2015) Characterizations of input-to-state stability for systems with multiple invariant sets. IEEE Transactions on Automatic Control

Angeli D, Sontag ED, Wang Y (2003) Input-to-state stability with respect to inputs and their derivatives. Int $\mathrm{J}$ Robust Nonlinear Control 13(11):1035-1056

Bastin G (1999) Issues in modelling and control of mass balance systems. In: Stability and stabilization of nonlinear systems, Springer, pp 53-74 
Beisner B, Haydon D, Cuddington K (2003) Alternative stable states in ecology. Front Ecol Environ 1(7):376-382. https://doi.org/ 10.1890/1540-9295(2003)001[0376:ASSIE]2.0.CO;2

Braun M (1993) Differential equations and their applications, Texts in applied mathematics, vol 11, fourth edition edn. Springer. https://doi.org/10.1007/978-1-4612-4360-1

Chaves M (2005) Input-to-state stability of rate-controlled biochemical networks. SIAM J Control Optim 44(2):704-727

Chaves M, Eissing T, Allgöwer F (2008) Bistable biological systems: a characterization through local compact input-to-state stability. IEEE Trans Autom Control 53(Special Issue):87-100

Coleman K, Jenkinson D (1999) Rothc-26.3 a model for the turnover of carbon in soil: model description and windows user guide (modified 2008). Tech. rep., IACR Rothamsted

Cushing J, Constatino R, Dennis B, Desharnais R, Henson S (2003) Chaos in ecology: experimental nonlinear dynamics. Academic Press theoretical ecology series, Academic Press. https://books. google.de/books?id=pzwIFeFZt6sC

Edwards HA, Lin Y, Wang Y (2000) On input-to-state stability for time varying nonlinear systems. In: Proceedings of the 39th IEEE conference on decision and control, 2000. IEEE, vol 4, pp 35013506

Feinberg M (1977) Mathematical aspects of mass action kinetics. Chemical reactor theory: a review, pp. 1-78

Feinberg M (1987) Chemical reaction network structure and the stability of complex isothermal reactors-I. The deficiency zero and deficiency one theorems. Chem Eng Sci 42(10):2229-2268

Fontaine S, Barot S (2005) Size and functional diversity of microbe populations control plant persistence and longterm soil carbon accumulation. Ecol Lett 8(10):1075-1087. https://doi.org/10.1111/j.1461-0248.2005.00813.x

Grimm V, Wissel C (1997) Babel, or the ecological stability discussions: an inventory and analysis of terminology and a guide for avoiding confusion. Oecologia 109(3):323-334. https://doi.org/10.1007/s004420050090

Guckenheimer J, Holmes P (1983) Nonlinear oscillations, dynamical systems, and bifurcations of vector fields, Applied mathematical sciences, vol 42. Springer. https://doi.org/10.1007/978$1-4612-1140-2$

Hearon JZ (1953) The kinetics of linear systems with special reference to periodic reactions. Bull Math Biophys 15(2):121-141

Hearon JZ (1963) Theorems on linear systems. Ann N Y Acad Sci 108(1):36-68

Henin S, Dupuis M (1945) Essai de bilan de la matière organique du sol. Annales Agronomique 15:17-29

Henin S, Monnier G, Turc L (1959) Un aspect de la dynamique des matieres organiques du soi. Comptes rendus hebdomadaires des séances de l'Académie des sciences 248(1):138-141

Hoang H, Couenne F, Jallut C, Le Gorrec Y (2011) The port Hamiltonian approach to modeling and control of continuous stirred tank reactors. J Process Control 21(10):1449-1458

Holling CS (1973) Resilience and stability of ecological systems. Annu Rev Ecol Syst 4:1-23

Horn F, Jackson R (1972) General mass action kinetics. Arch Ration Mech Anal 47(2):81-116

Jacquez JA, Simon CP (1993) Qualitative theory of compartmental systems. Siam Review 35(1):43-79

Kloeden P, Rasmussen M (2011) Nonautonomous dynamical systems. American Mathematical Society

Kot M (2001) Elements of mathematical ecology. Elements of mathematical ecology. Cambridge University Press, Cambridge. https:// books.google.de/books?id=7_IRInNON7oC

Lenton TM, Held H, Kriegler E, Hall JW, Lucht W, Rahmstorf S, Schellnhuber HJ (2008) Tipping elements in the Earth's climate system. Proceedings of the National Academy of Sciences
105(6):1786-1793. https://doi.org/10.1073/pnas.0705414105. http:// www.pnas.org/content/105/6/1786.abstract, http://www.pnas.org/ content/105/6/1786.full.pdf+html

Liu S-J, Zhang J-F, Jiang Z-P (2008) A notion of stochastic input-tostate stability and its application to stability of cascaded stochastic nonlinear systems. Acta Math Appl Sin Eng Ser 24(1):141-156

Ludwig D, Jones DD, Holling CS (1978) Qualitative analysis of insect outbreak systems: the spruce budworm and forest. J Anim Ecol 47(1):315-332. https://doi.org/10.2307/3939

Luo Y, Weng E (2011) Dynamic disequilibrium of the terrestrial carbon cycle under global change. Trends Ecol Evol 26(2):96-104

Luo Y, Keenan TF, Smith M (2015) Predictability of the terrestrial carbon cycle. Glob Chang Biol 21(5):1737-1751. https://doi.org/10.1111/gcb.12766

Manzoni S, Porporato A (2007) A theoretical analysis of nonlinearities and feedbacks in soil carbon and nitrogen cycles. Soil Biol Biochem 39(7):1542-1556. https://doi.org/10.1016/j. soilbio.2007.01.006

Manzoni S, Katul GG, Porporato A (2009) Analysis of soil carbon transit times and age distributions using network theories. J Geophys Res Biogeo 114(G4):g04025. https://doi.org/10. 1029/2009JG001070

Maschke B, Ortega R, Van Der Schaft AJ (2000) Energy-based Lyapunov functions for forced Hamiltonian systems with dissipation. IEEE Trans Autom Control 45(8):1498-1502

May RM (1973) Qualitative stability in model ecosystems. Ecology 54(3):638-641. https://doi.org/10.2307/1935352

Maynard-Smith J (1978) Models in ecology. Cambridge University Press, Cambridge. https://books.google.de/books?id= Jgk4AAAAIAAJ

Meyer K (2016) A mathematical review of resilience in ecology. Nat Resour Model 29(3):339-352

Mulholland RJ, Keener MS (1974) Analysis of linear compartment models for ecosystems. J Theor Biol 44(1):105-116. https://doi.org/10.1016/S0022-5193(74)80031-7

Nolting BC, Abbott KC (2016) Balls, cups, and quasi-potentials: quantifying stability in stochastic systems. Ecology 97(4):850-864. https://doi.org/10.1890/15-1047.1

Olson JS (1963) Energy storage and the balance of producers and decomposers in ecological systems. Ecology 44(2):322-331

Parton WJ, Schimel DS, Cole CV, Ojima DS (1987) Analysis of factors controlling soil organic matter levels in great plains grasslands. Soil Sci Soc Am J 51(5):1173-1179. https://www.soils.org/ publications/sssaj/abstracts/51/5/1173

Pastor J (2008) Mathematical ecology of populations and ecosystems. Wiley-Blackwell. https://books.google.de/books? id=PzfkRCN4wegC

Pimm SL (1984) The complexity and stability of ecosystems. Nature 307(5949):321-326. http://dx.doi.org/10.1038/307321a0

Rasmussen M, Hastings A, Smith MJ, Agusto FB, Chen-Charpentier BM, Hoffman FM, Jiang J, Todd-Brown KEO, Wang Y, Wang YP, Luo Y (2016) Transit times and mean ages for nonautonomous and autonomous compartmental systems. J Math Biol 73:1379-1398. https://doi.org/10.1007/s00285-016-0990-8

Raupach MR (2013) The exponential eigenmodes of the carbon-climate system, and their implications for ratios of responses to forcings. Earth Syst Dynam 4(1):31-49. https://doi.org/10.5194/esd-4-31-2013

Scheffer M, Carpenter SR (2003) Catastrophic regime shifts in ecosystems: linking theory to observation. Trends Ecol Evol 18(12):648656. https://doi.org/10.1016/j.tree.2003.09.002. http://www. sciencedirect.com/science/article/pii/S0169534703002787

Scheffer M, Carpenter S, Foley JA, Folke C, Walker B (2001) Catastrophic shifts in ecosystems. Nature 413(6856):591-596. https://doi.org/10.1038/35098000 
Schimel JP, Weintraub MN (2003) The implications of exoenzyme activity on microbial carbon and nitrogen limitation in soil: a theoretical model. Soil Biol Biochem 35(4):549-563. https://doi.org/10.1016/S0038-0717(03)00015-4

Sierra CA, Müller M (2015) A general mathematical framework for representing soil organic matter dynamics. Ecol Monogr 85(4):505-524. https://doi.org/10.1890/15-0361.1

Sinsabaugh RL, Follstad Shah JJ (2012) Ecoenzymatic stoichiometry and ecological theory. Annual Review of Ecology, Evolution, and Systematics. https://doi.org/10.1146/annurev-ecolsys071112-124414

Soetaert K, Herman P (2009) A practical guide to ecological modelling. Springer, Netherlands. https://doi.org/10.1007/978-14020-8624-3_1

Sontag E (2008) Input to state stability: basic concepts and results. In: Nistri P, Stefani G (eds) Nonlinear and optimal control theory, Lecture notes in mathematics, vol 1932, Springer Berlin Heidelberg, pp 163-220. https://doi.org/10.1007/978-3-540-77653-6_3

Sontag ED (1989) Smooth stabilization implies coprime factorization. IEEE Trans Autom Control 34(4):435-443

Sontag ED (1998a) Comments on integral variants of ISS. Syst Control Lett 34(1):93-100

Sontag ED (1998b) Mathematical control theory: deterministic finite dimensional systems, Textbooks in applied mathematics, vol 6 , 2nd edn. Springer Science \& Business Media

Sontag ED (2001) Structure and stability of certain chemical networks and applications to the kinetic proofreading model of T-cell receptor signal transduction. IEEE Trans Autom Control 46(7):1028-1047

Sontag ED, Wang Y (1995) On characterizations of the input-to-state stability property. Syst Control Lett 24(5):351-359

Sontag ED, Wang Y et al (1996) New characterizations of input-tostate stability. IEEE Trans Autom Control 41(9):1283-1294
Strogatz SH (1994) Nonlinear dynamics and chaos: with applications to physics, biology, chemistry, and engineering. Perseus publishing

Tsinias J (1998) Stochastic input-to-state stability and applications to global feedback stabilization. Int J Control 71(5):907-930

Tsinias J, Karafyllis I (1999) ISS property for time-varying systems and application to partial-static feedback stabilization and asymptotic tracking. IEEE Trans Autom Control 44(11):21792184

Wang G, Post WM, Mayes MA (2012) Development of microbialenzyme-mediated decomposition model parameters through steady-state and dynamic analyses. Ecol Appl 23(1):255-272. https://doi.org/10.1890/12-0681.1

Wang G, Post WM, Mayes MA (2013) Development of microbialenzyme-mediated decomposition model parameters through steady-state and dynamic analyses. Ecol Appl 23(1):255-272. https://doi.org/10.1890/12-0681.1

Wieczorek S, Ashwin P, Luke CM, Cox PM (2011) Excitability in ramped systems: the compost-bomb instability. Proceedings of the Royal Society of London A: Mathematical, Physical and Engineering Sciences 467(2129):1243-1269. https://doi.org/10.1098/rspa. 2010.0485. http://rspa.royalsocietypublishing.org/content/467/ 2129/1243, http://rspa.royalsocietypublishing.org/content/467/ 2129/1243.full.pdf

Xia J, Luo Y, Wang YP, Hararuk O (2013) Traceable components of terrestrial carbon storage capacity in biogeochemical models. Glob Chang Biol 19(7):2104-2116. https://doi.org/10. $1111 /$ gcb. 12172

Zelenev V, van Bruggen A, Semenov A (2000) BACWAVE, a spatial-temporal model for traveling waves of bacterial populations in response to a moving carbon source in soil. Microb Ecol 40(3):260-272. https://doi.org/10.1007/s002480000029 